\title{
Uncertainty analysis of a WEC model test experiment
}

\author{
Jarrah Orphin ${ }^{1,2}$, Jean-Roch Nader ${ }^{1}$, Irene Penesis $^{1}$ \\ ${ }^{1}$ National Centre for Maritime Engineering and Hydrodynamics, Australian Maritime College, University of Tasmania \\ ${ }^{2}$ Bombora Wave Power, Cleddau Reach, Pembroke Dock, SA726UJ, Wales, UK. jarrah.orphin@bomborawave.com
}

5 Abstract

Ocean wave energy has significant technical potential but limited full-scale deployments and technology convergence. Consequently, guidelines for developing wave energy converters (WECs) are still developing themselves, especially around experimental uncertainty analysis (UA). To develop a comprehensive WEC-specific UA methodology, we conducted a 1:30 scale experiment of a case study oscillating water column (OWC) WEC. This paper presents the methodology, which describes UA principles and means to identify parameters causing uncertainty, and demonstrates new WEC-specific UA methods: general uncertainty analysis (GUA), the Monte Carlo method (MCM) for uncertainty propagation, and Type A and Type B uncertainty evaluation. Results show the expanded uncertainty averaged $\pm 16 \%$ for capture width ratio and $\pm 6 \%$ for wave loads; Type $\mathrm{B}$ uncertainty tended to be slightly larger than Type A uncertainty; and uncertainty in regular waves slightly larger than irregular waves. The MCM was found to be effective and efficient in uncertainty propagation. In general, given WECs tend to maximise motions, use a power take-off system, and must survive storms, WEC model tests may be especially susceptible to uncertainty due to nonlinearities and modelling complexities. In conclusion, UA should be carried out in WEC model test experiments. We close with recommendations for refining relevant international guidelines.

Keywords: Uncertainty analysis, wave energy converter, experiment

\section{Introduction}

Guidelines and standards support the development of technologies from idea to prototype to product, so they must be accurate, robust, and meet industry needs. In ocean wave energy, which has significant technical potential [1, guidelines for testing and developing wave energy converter (WEC) technologies have been established by research projects [2, 3, 4, 5, 6, 7, and, more recently, by international organisations [8, 9]. These guidelines, however, are still undergoing active development because of limited full-scale deployments that feedback knowledge and because of the large diversity of WECs still being developed [10, 11. In particular, guidelines are lacking on uncertainty analysis (UA) in hydrodynamic model test experiments of WECs [12. This lack is important to address because WEC 
technology developers, researchers, consultants, and investors make critical decisions based on model test results: proof-of-concept, validation and calibration of numerical models, system identification, design and prediction of prototype performance, to inform Levelised Cost of Energy (LCOE), and investment [13, 9, 7].

UA is key to understanding the causes and effects of uncertainty in model test experiments. Unfortunately, UA is neither well understood nor widely used in WEC model tests [12. Despite sound knowledge of UA in mature maritime fields such as general hydrodynamic experimentation [14], offshore structures [15], ships [16, 17, and coastal engineering [18, applying this knowledge in wave energy, particularly in WEC experiments, is inadequate and carries risk because WECs tend to maximise motions, use a power take-off (PTO) system, often have novel geometries and moorings that influence motions, and are exposed to extreme wave-induced loads [13. The few studies that have focused on the problem of experimental uncertainty in WEC model tests show that, in consequence of these unique WEC characteristics, uncertainty can considerably influence experimental results [19, 20, 21, 22]. For example, spatial variations of generated waves in wave tanks [19 coupled with difficult to measure interactions and absorbed power of an array of heaving-buoy WECs lead to uncertainty levels that mostly concealed motions and power [20]. Capture width ratio of oscillating water column (OWC) WECs has also been shown to be especially susceptible to uncertainty [21, 22. More broadly, uncertainty extends beyond the laboratory to uncertainty in wave resource assessment [23, 24, 25, mean annual energy production (MAEP) estimates [25, 26], and open water tests [27.

To address the lacking WEC-specific knowledge of experimental uncertainty, guidelines have been developed in research projects [6, 5] and by the International Towing Tank Conference (ITTC) with Uncertainty Analysis for a Wave Energy Converter 7.5-02-07-03.12 28]. While these are a good step toward rigorous, standardised best practices, the guidance is relatively immature; it lacks important uses of UA, such pre-test or general uncertainty analysis, alternative methods to propagate uncertainty such as the Monte Carlo method (MCM) 29, 30, 22], and WEC-specific methods for evaluating Type A and Type B uncertainty [21]. To refine this guidance, we carried out a 1:30 scale experiment of a case study oscillating water column (OWC) WEC, based on Australian company Wave Swell Energy's Uniwave ( technology system enabled by passive valves. The aim was to develop a comprehensive UA methodology, that could be used as a template or guidance for most WEC designs and experiments - the work is thus necessarily descriptive.

This paper presents the methodology and demonstrates how UA was applied in the experiment. It is structured by seven experimental phases: planning, design, construction, debugging, execution,

\footnotetext{
${ }^{1}$ https://www.waveswell.com/technology/
} 
analysis, and reporting (based on [30]). It introduces to the wave energy field the concepts of general uncertainty analysis (GUA), used in experiment planning and design, and detailed uncertainty analysis (DUA), used in subsequent experimental phases. It also develops the MCM for use in both GUA and DUA to effectively and efficiently propagate uncertainty in nonlinear and time-dependent measurands (capture width ratio and its lower-level measurands and quantities). Step by step, the principles of GUA and DUA are explained and reinforced by rich examples of their application in the experiment, including power performance and wave loads results from the experiment that is representative of technology readiness level (TRL) 1-4. Ultimately, the work argues for the importance of UA, that it is worthwhile investing in understanding it and using it in WEC model test experiments.

\section{Overview of experimentation and uncertainty analysis}

UA is the analysis of uncertainties in an experiment to assure and quantify the quality of results.

75 Figure 1 shows the relation between UA uses and experimentation phases and activities. UA is split into two categories: GUA, used in the planning and initial design phase of an experiment, and DUA, used in the remaining experimental phases of design, construct, debug, execute, analyse, and report [30].

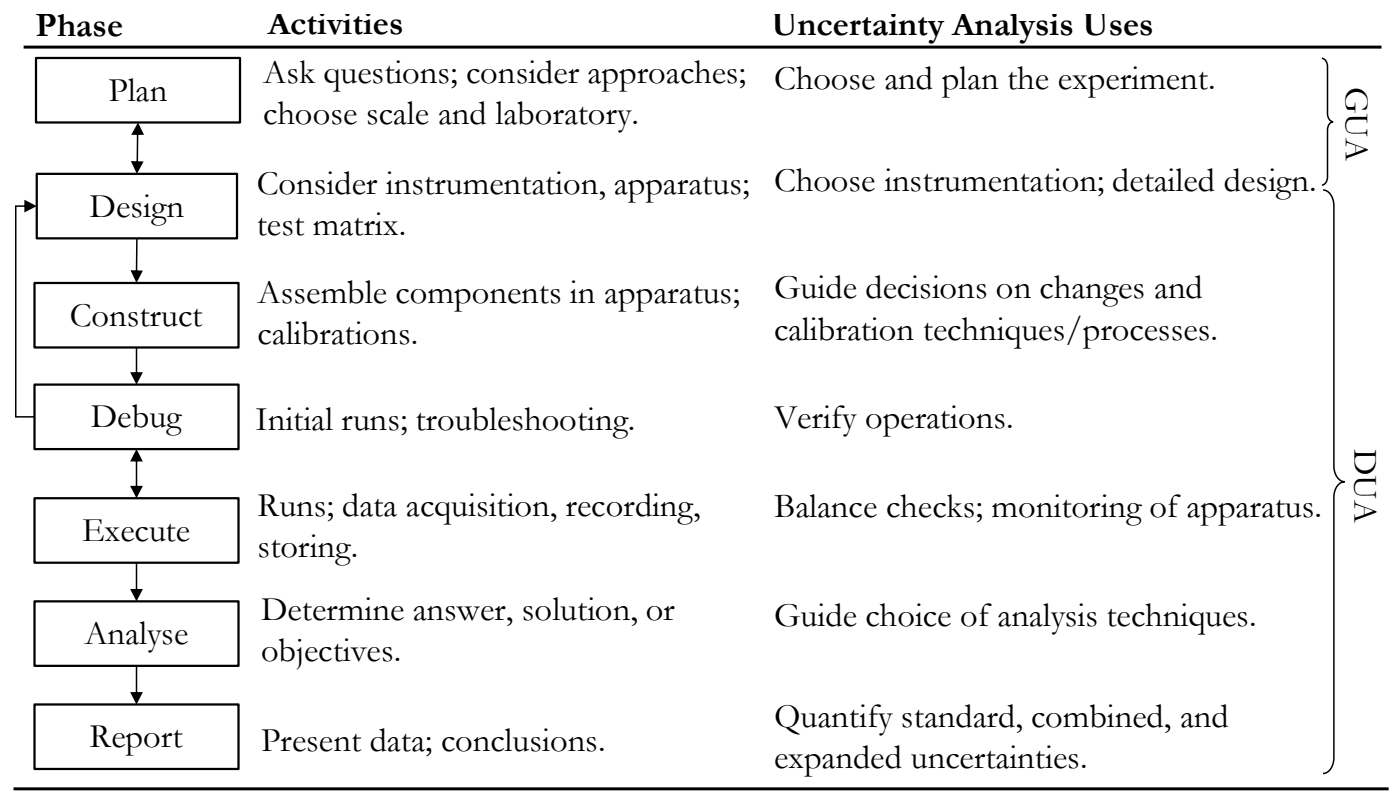

Figure 1: Experimental phases with descriptive activities and corresponding uses of uncertainty analysis.

GUA requires evaluating the relative importance of general uncertainties in an experiment. In particular, it requires evaluating various approaches, instruments, apparatus, and measurement procedures that might best answer the questions or objectives of interest. DUA, on the other hand, 
requires evaluating uncertainties at a detailed level, to guide decisions on experimental design and implementation, to monitor data and its uncertainty, and to quantify the quality of results throughout the experiment. To provide a systematic means of performing both GUA and DUA, we introduce a three-stage structure: (1) formulate, (2) propagate, and (3) summarise (based on [31, 29]). Figure 2 shows the concepts in and process of these stages.

Descriptions of key terms in Figure 2 are as follows:

- Measurand model: $Y=f\left(X_{1}, X_{2}, \ldots, X_{N}\right)$, where $Y$ is the output quantity and is a function of $i=1: N$ number of input quantities $X_{i}$ (lowercase $x_{i}, y$ are the estimates or measurements of quantities)

- PDF: probability density function of input quantities $X_{i}$

- $u_{G}\left(X_{i}\right)$ : general uncertainty, one value characterised by an assumed PDF of $X_{i}^{\prime}$ 's

- $u_{A}\left(x_{i}\right)$ : Type A uncertainty - evaluated by statistical analysis, i.e., the standard deviation of the PDF produced by repeated observations

- $u_{B}\left(x_{i}\right)$ : Type B uncertainty - evaluated from instrument calibrations, manufacturer's specifications, or uncertainties taken from handbooks

\section{Case study WEC description}

The case study WEC was based on the Wave Swell Energy (WSE) Uniwave technology, which is a bottom-fixed oscillating water column (OWC) WEC (Figure 3). Unlike conventional OWC WECs, which use bidirectional or rectified flow PTO systems [32, the WSE technology uses valves to create a unidrectional flow PTO system. This PTO comprises a unidirectional nonlinear air turbine, with unidirectional airflow enabled by valves that open and exhaust air on the up-stroke of the inner free surface, then close on the down-stroke to direct air through the air turbine, thereby generating electricity (see [33] for more details).

Froude scaling was applied to design a 1:30 scale model of the WSE prototype WEC. The PTO system was modelled using an orifice plate to represent the quadratic air pressure-flow characteristics of the air turbine (a common practice [32, 34). Light weight, robust plastic sheets simulated the passive valves. subsection 5.6 further details the experimental design and physical model.

\section{General uncertainty analysis}

GUA is carried out before an experiment begins, and has many uses: it enforces us to examine an intended experiment and provides an integrated grasp of how to carry it out; identifies potential 


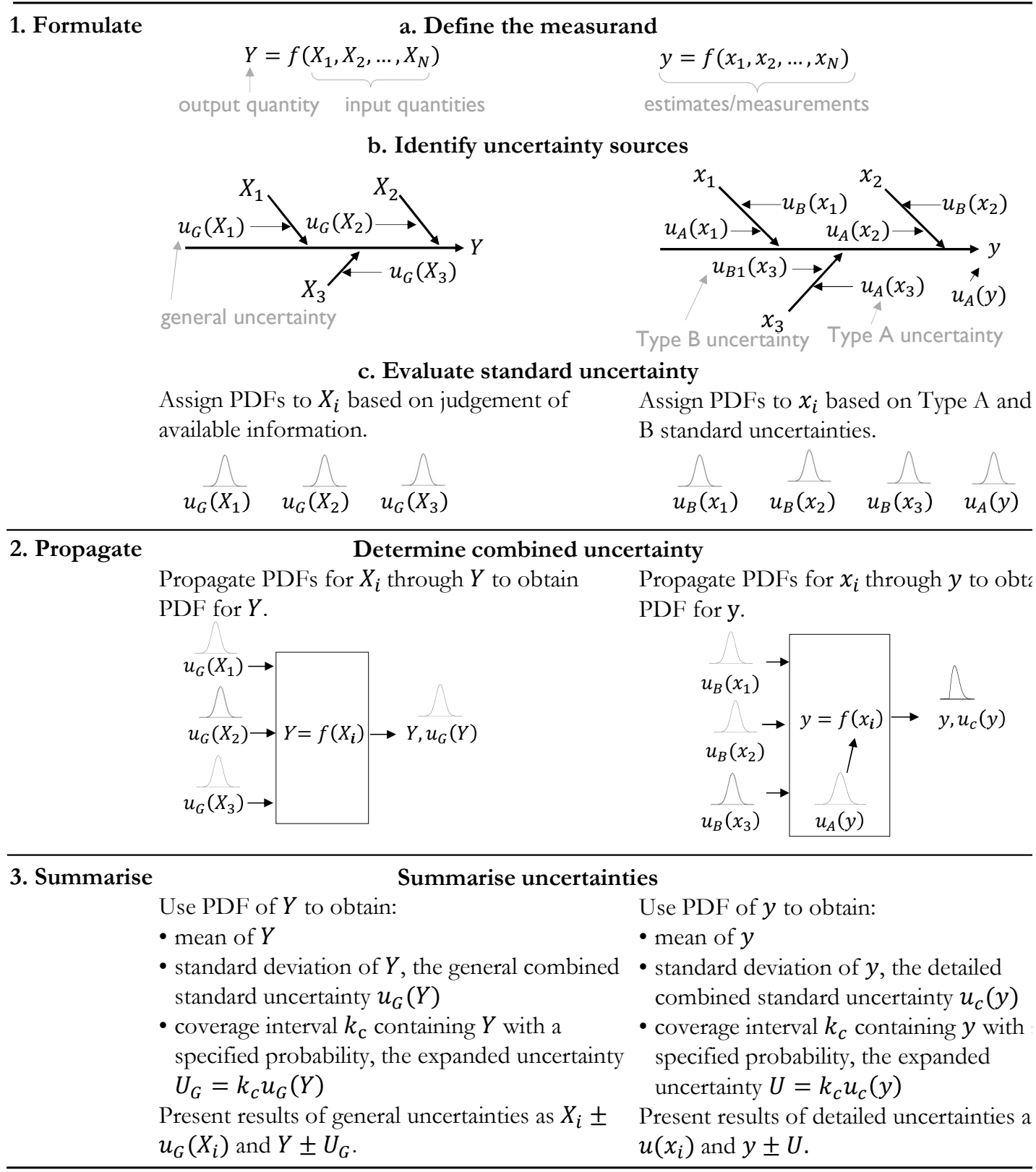

Figure 2: Overview of the concepts and processes of uncertainty analysis, structured by three main stages, for General Uncertainty Analysis and Detailed Uncertainty Analysis. The example illustrates $N=3$ independent input quantities $X_{i}$.

troubles and errors, and why they exist; advises when improved instruments or procedures are needed to reduce uncertainty, while minimising cost; and reveals which parameters contribute most to the uncertainty in results, thereby focusing attention on critical measurements and key procedures which govern the overall experimental uncertainty [36]. All these uses permit access to otherwise inaccessible 

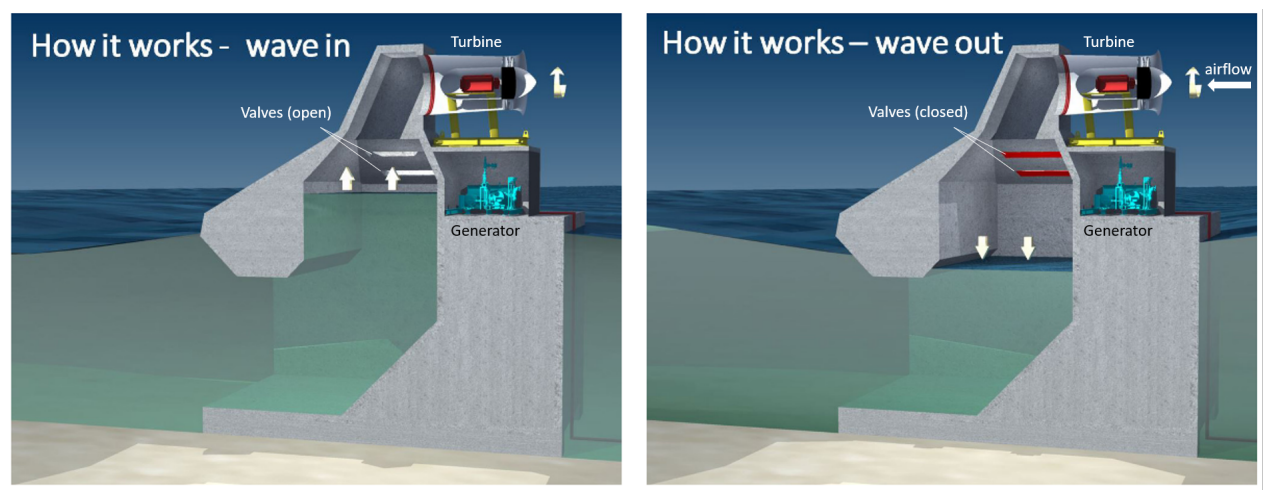

Figure 3: Working principle of the Wave Swell Energy Oscillating Water Column Wave Energy Converter, the case study technology used as the subject of this research (prototype shown, adapted from [35]).

information about an experiment before it begins, saving time, money, and resources [30]. This section describes the principles of GUA and our application of it, according to the three-stage UA structure presented in Figure 2.

\subsection{Stage 1a. Formulate: Define the measurand}

To define the measurand(s) of the experiment is to describe, mathematically, what is being measured. In most cases a measurand $Y$ is not measured directly but is a measurement model: $Y=$ $f\left(X_{1}, X_{2}, \ldots, X_{N}\right)$ (the $X_{i}$ 's may themselves be lower-level measurands). For this experiment, to define the measurands a simple sketch of the intended experiment was created (Figure 4). The sketch shows the processes and parameters of the experiment. $X_{i}$ 's are assembled into the measurand functions (boxed equations). The following equations describe the mathematical model of incident waves, the WEC, and their interaction.

Beginning with incident waves, assuming linear wave theory with regular long-crested two-dimensional waves of elevation $\eta_{i n c}$, angular frequency $\omega$, and water depth $h$, propagating toward the OWC WEC in a constant direction, the regular wave power $P_{W_{r}}$ per unit length in general water depth is

$$
P_{W_{r}}=\frac{1}{8} \rho_{w} g H^{2} c_{g}
$$

where $H$ is the incident wave height, $\rho_{w}$ the water density, $g$ the gravitational acceleration, and $c_{g}$ the group velocity. Group velocity $c_{g}$ is calculated from wave celerity $c$ and wave number $k$,

$$
c_{g}=\frac{c}{2}\left[1+\frac{2 k h}{\sinh (2 k h)}\right]
$$

where

$$
c=\frac{\omega}{k}
$$




$$
P=\frac{1}{T} \int p q d T \quad C_{W}=\frac{P}{P_{W_{\text {reg,irr }} B}}
$$

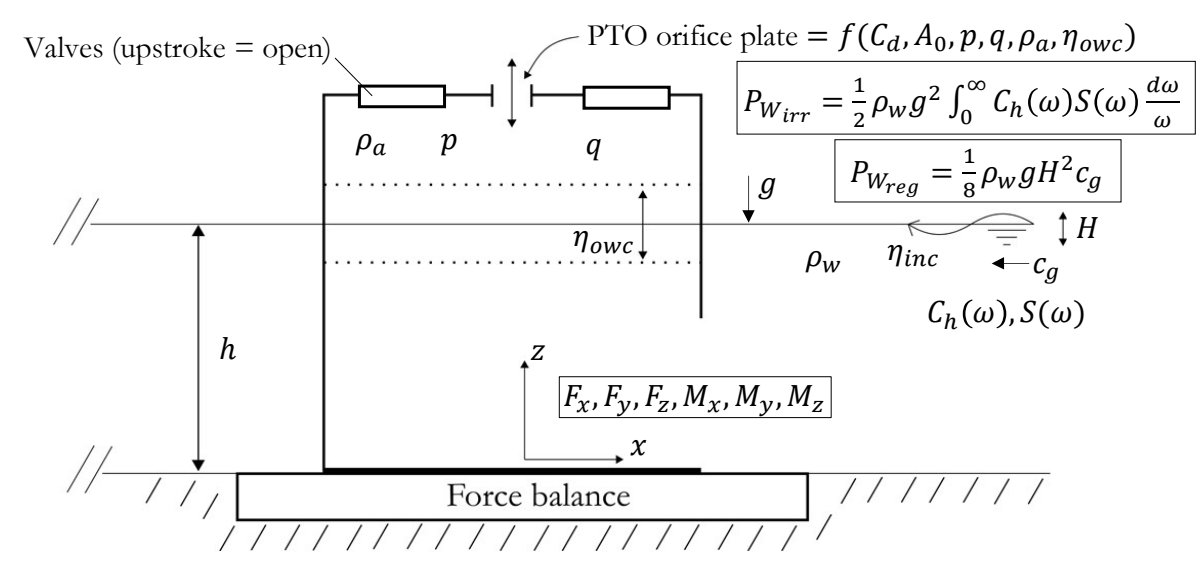

Figure 4: Simple sketch of the OWC WEC experiment in regular waves, showing all the defined measurands and input quantities on which they depend.

with $k$ determined through an iterative process by solving the dispersion relationship

$$
\omega^{2}=g k \tanh (k h)
$$

The total average irregular wave power $P_{W_{i}}$ in finite water depth for a given spectrum is

$$
P_{W_{i}}=\frac{1}{2} \rho g^{2} \int_{0}^{\infty} C_{h}(\omega) S(\omega) \frac{d \omega}{\omega}
$$

135

where $S(\omega)$ is the power spectral density function and $C_{h}(\omega)$ is a correction that modifies the wave power in deep water to wave power in finite water depth [37:

$$
C_{h}=\left(1+\frac{2 k h}{\sinh (2 k h)}\right) \frac{k_{0}}{k}
$$

where $k_{0}=\omega^{2} / g$ is the wave number in deep water $\left(h>\frac{1}{2} \lambda\right)$. For a given spectrum, the significant wave height $H_{m 0}$ is

$$
H_{m 0}=4 \sqrt{m_{0}}
$$

and various wave period statistics of energy period $T_{e}$, zero up-crossing period $T_{z}$, and mean wave period $T_{m 01}$ are respectively:

$$
T_{e}=\frac{m_{-1}}{m_{0}}, T_{z}=\sqrt{\frac{m_{0}}{m_{2}}}, T_{m 01}=\frac{m_{1}}{m_{0}}
$$

with the spectral moments defined as

$$
m_{n}=\int_{0}^{\infty} S(\omega) \omega^{n} d \omega
$$


where $n=-1,0,1,2, \ldots$

Pneumatic power $P$, henceforth 'OWC power', is derived from the differential air pressure $p$ measured inside the OWC chamber, and air volume flow rate $q$ displaced by the motion of the inner free-surface. In regular waves,

$$
P=\frac{1}{T} \int p q d T
$$

where $T$ is the characteristic wave period. There are two key methods of deriving $q$ in Equation 10 subsection 4.6 describes these methods and presents an example of how GUA was used to choose which method would result in the least uncertainty.

$P$ in irregular waves is the same as Equation 10 except $T$ is the time vector of the whole irregular wave timeseries.

Capture width ratio $C_{W}$ is the top-level measurand that relates $P$ to $P_{W}$ as a function of the characteristic width $B$ of the WEC:

$$
C_{W}=\frac{P}{P_{W_{r, i}} B}
$$

It is worth noting that although $P_{W}$ assumes linear waves, even it is not always the case, it is mostly used to non-dimensionalise of $P$, whereas the calculation of $P$ includes nonlinearities.

Figure 4 also shows hydrodynamic wave loads imposed on the OWC WEC, to be measured using a six-component force balance. The six load measurands are surge force $F_{x}$, sway force $F_{y}$, heave force $F_{z}$, roll moment $M_{x}$, pitch moment $M_{y}$, and yaw moment $M_{z}$. This work focuses only the important components of $F_{x}, F_{z}$, and $M_{y}$.

\subsection{Stage 1b. Formulate: Identify uncertainty sources}

There are many possible sources of measurement uncertainty (see 31 for a generic list). A broader perspective on experimental uncertainty reveals yet more uncertainties (Table 1). All uncertainties should be considered and the relevant ones evaluated. Cause-and-effect diagrams are useful here

(Figure 5) 38. These diagrams effectively account for the general uncertainties $u_{G}\left(X_{i}\right)$ in the $X_{i}$ 's and show their relative influences on the overall uncertainty in $Y$.

\subsection{Stage 1c. Formulate: Evaluate standard uncertainty}

To evaluate standard uncertainty requires assigning PDFs - Gaussian (normal), rectangular (uniform), etc. - to the $X_{i}$ on the basis of available knowledge, and assigning instead a joint PDF to those $X_{i}$ that are not independent [31. Available knowledge can include previous measurement data, experience with similar experiments, or reference materials. Often a simple normal PDF is assigned at this pre-experiment stage [30. 
Table 1: Potential sources of uncertainty in a typical wave energy converter experiment.

\section{Parameter}

Scaling

Instrumentation

Laboratory

Fluid properties

Data reduction and analysis

Human factors

\section{Uncertainties}

Non-similitude of Froude and Reynolds numbers; structural materials; PTO simplification; air compressibility; nonlinear waves; water depth truncation; sharp corners and narrow funnels on models [18, 15, 39, 9, 40.

Geometry, articulations; construction; installation; hydrostatics; moorings; PTO (friction, limitations, control); elasticity/nonlinear deformations [15, 28, 9].

nonlinearity, hysteresis, calibration, noise; sensor positions; influence on model motions [15, 41].

Wave generation/control (higher-order waves, transverse non-uniformity, inputmeasured discrepancy); boundaries (wave reflections, blockage); limited run durations; initial conditions (residual waves, turbulence) [18, 15, 28].

Viscosity; density (change in buoyancy, mass distribution, pressure/forces, temperature; surface tension; wave breaking [18, 42, 15, 43].

Model definition, idealisation, assumptions; test definition; propagation of uncertainty for measurands [4, 30].

Experiment setup; calibrations; judgement under uncertainty; measurement reading error; data analysis and reporting [15, 45].
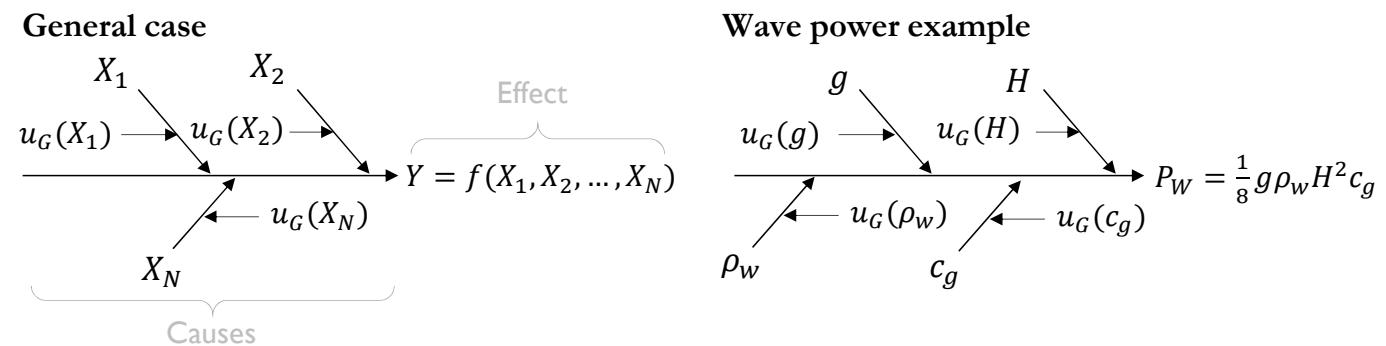

Figure 5: A cause-and-effect diagram used to identify uncertainty sources in a measurand $Y$, where $X_{i}$ are input or influence quantities upon which $Y$ depends, and $u_{G}\left(X_{i}\right)$ is a general uncertainty associated with $X_{i}$. Example is for $N=3$ independent $X_{i}$.

\subsection{Stage 2. Propagate: Determine combined uncertainty}

To determine combined uncertainty requires uncertainty propagation, whereby the PDFs of the $X_{i}$ are propagated through a measurand function to produce an estimate and PDF of $Y$. Propagating PDFs can be implemented either analytically, using the 'law of propagation of uncertainty' based on a first-order Taylor series approximation (i.e., the Taylor Series Method (TSM) or GUM uncertainty 
framework [31]), or statistically by performing random sampling from PDFs using, for example, the Monte Carlo method (MCM) [29, 30. The MCM is a practical alternative to the TSM. It has value when linearisation of the measurand provides an inadequate representation, or the PDF for $Y$ is nonlinear and departs appreciably from a normal PDF or a scaled and shifted t-distribution. Further, the MCM directly propagates uncertainty through measurand functions and so does not contain approximations (therefore errors) which the TSM does; often reduces the analysis effort; and is more reliable when an estimate of $Y$ and its associated uncertainty are approximately of the same magnitude. For these reasons the MCM has become the primary method for uncertainty propagation in many engineering fields [30]. The MCM, therefore, is the focus of this work. It is especially useful in this application, and probably most WEC experiments, because the experiment is characterised by multiple time-dependent nonlinear processes - nonlinear waves inducing nonlinear motions of the OWC internal free surface which interacts with a nonlinear PTO.

Figure 6 shows the process of the MCM in GUA, presenting an example where a measurand $Y$ depends on two input quantities $X_{1}$ and $X_{2}$ such that $Y=f\left(X_{1}, X_{2}\right)$. The method, however, is general for measurands with any number of inputs. A description of the method and its implementation follows.

First, assumed nominal values are input for each quantity $X_{1, n o m}$ and $X_{2, n o m}$, as well as their assumed general uncertainties $u_{G}\left(X_{1}\right)$ and $u_{G}\left(X_{2}\right)$. Each $u_{G}$ is a unique value encompassing uncertainty components, and is assumed to be the standard deviation of the assumed PDF, which is typically normal but other distributions can be assumed based on better knowledge. Then, at each iteration $\mathrm{j}$, $u_{G}\left(X_{i}\right)$ is multiplied by a randomly sampled number drawn from the assumed PDF (varying about 1), and added to the nominal values of each quantity to obtain the "measured" values $X_{1}(\mathrm{j})$ and $X_{2}(\mathrm{j})$. From these measured values the result of the measurand $Y$ is calculated. This sampling process is repeated $M$ times to obtain a PDF for $Y$. The output of the MCM is the standard deviation of the PDF of $Y$, taken as the general uncertainty $u_{G}(Y)$. An appropriate value for $M$ is determined by calculating the standard deviation of $Y$ at each iteration and stopping the process when a converged value is reached ([22]). A converged value to within $5 \%$ is considered to give an acceptable approximation of $u_{G}(Y)(M \approx 5,000$ is typically sufficient $)$.

At this pre-experiment stage, a sensitivity analysis may be performed through a series of simulations over a range of nominal values and uncertainties within the expected parameter space of the experiment. Here, one input quantity is subject to random sampling at a time, while the other quantities remain constant. The analysis reveals the relative importance of $u_{G}\left(X_{i}\right)$ 's on $u_{G}(Y)$, thereby yielding insights into which parameters contribute most to the overall uncertainty, valuable information that informs the subsequent experiment design phase. 


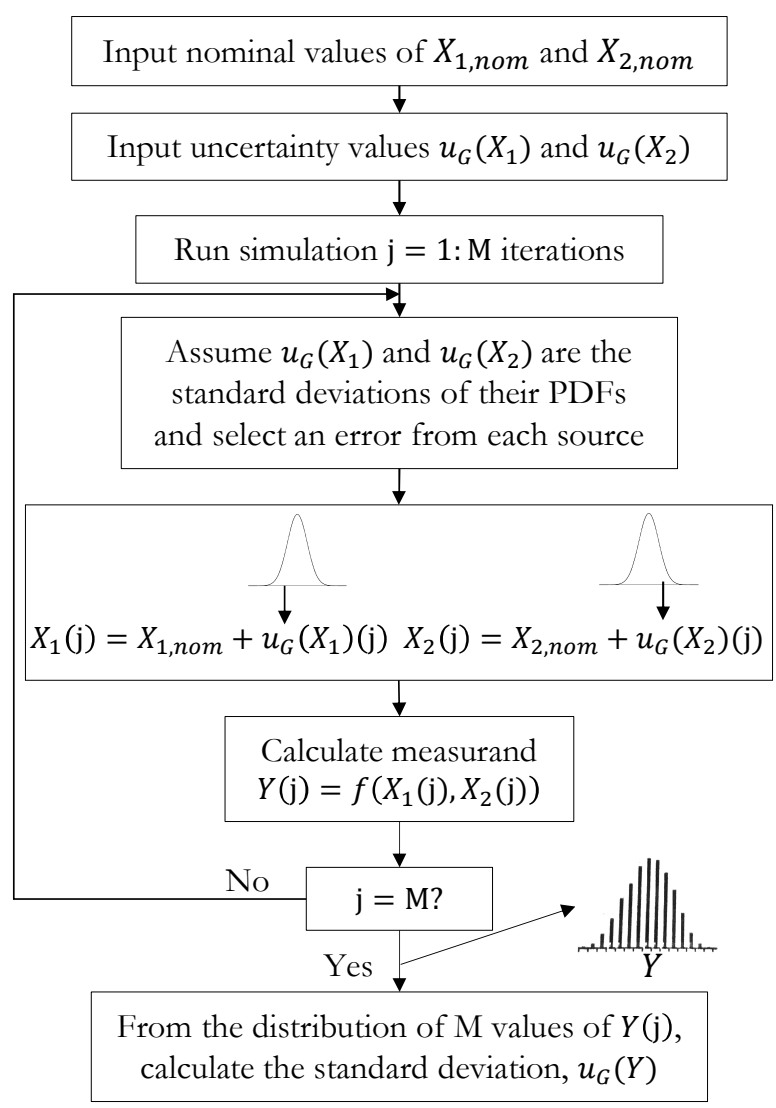

Figure 6: Flow diagram of the Monte Carlo method to propagate uncertainty in general uncertainty analysis, showing an example where the measurand result $Y$ is a function of two input quantities $X_{1}$ and $X_{2}$.

\subsection{Stage 3. Summarise: Summarise uncertainties}

To summarise uncertainties requires using the PDF for $Y$ to obtain: an estimate (the mean) of $Y$; the standard deviation of $Y$, taken as the general uncertainty $u_{G}(Y)$ associated with $Y$; and a coverage interval $k$ containing $Y$ with a specified probability, which gives the expanded general uncertainty $U_{G}=k u_{G}(Y)$ (Figure 2). To facilitate experiment planning and subsequent phases, these estimates and uncertainties should be presented in tables or graphs in the form $X_{i} \pm u_{G}\left(X_{i}\right)$ and $Y \pm U_{G}$.

The procedure for determining an MCM coverage interval for $Y$, even if the $\mathrm{PDF}$ is asymmetric, is as follows. First, sort the vector of $Y$ values that form the PDF from smallest to largest. Then, for a chosen coverage probability $p_{c}$, the lower bound of the coverage uncertainty interval is $Y_{\text {low }}=$ sorted vector $\left\{\left[\left(1-p_{c}\right) / 2\right] M\right\}$, with the upper bound $Y_{\text {high }}=$ sorted vector $\left\{\left[\left(1+p_{c}\right) / 2\right] M\right\}$. For example, to obtain $95 \%$ coverage interval limits $\left(p_{c}=0.95\right), Y_{\text {low }}=$ result number $(0.025 M)$ and $Y_{\text {high }}=$ result number $(0.975 M)$ (i.e., the $2.5 \%$ and $97.5 \%$ quantiles, with the interval containing $95 \%$ of the MCM results) 30 . 


\subsection{Example: Using the MCM to determine the best method to derive OWC power}

This example demonstrates how MCM was used in GUA to determine which out of two methods for deriving OWC power $P$ would result in the smallest uncertainty. It further reveals how the generated information informed the experimental design needed to achieve the estimated uncertainty. The example highlights how GUA can be especially helpful in determining whether or not a new procedure should be pursued in an experiment.

The problem of deriving $P$ is as follows. Referring to Equation 10, $P$ is a function of air pressure $p$ multiplied by volume flow rate $q$. Measuring $q$ in OWCs is challenging because the air flow changes continuously and rapidly, in both magnitude and sometimes direction. Therefore, $q$ is derived using two main methods [6]:

Method (a): $q$ is derived through a numerical derivation of air volume displaced from the OWC internal free surface $\eta_{\text {owc }}$ oscillations measured using multiple wave probes inside the OWC, hence $q_{\eta_{o w c}}:$

$$
q_{\eta_{\text {owc }}}=\iint_{S_{c}} \frac{d \eta_{o w c}}{d t} d s=\iint_{S_{c}} v_{s} d s
$$

where $S_{c}$ is the free surface area and $v_{s}$ the free surface velocity.

Method (b): $q$ is derived from measured $p$ and a calibrated orifice (used to simulate the PTO) characterised by a discharge coefficient $C_{d}$, hence $q_{C_{d}}$ :

$$
q_{C_{d}}=\frac{p}{|p|} C_{d} A_{0} \sqrt{\frac{2|p|}{\rho_{a}}}
$$

where $A_{0}$ is the orifice cross-sectional area, and $\rho_{a}$ the density of air, assumed to be $1.2 \mathrm{~kg} / \mathrm{m}^{3}$. This equation for deriving $q$ from flow through an orifice is according to ISO 5167 [4].

In-situ orifice calibration can be used to determine $C_{d}$, which is a two step process. First, we rearrange Equation 13 for $C_{d}$ and use data from all regular wave runs (either measurement data obtained during the experiment or data based on assumed values before the experiment) to calculate $C_{d}$ from values of $p, q_{C_{d}}, A_{0}$, and $\rho_{a}$. Obtaining $q_{C_{d}}$ here first requires the use of Method (a), such that $q_{C_{d}}=q_{\eta_{\text {owc }}}$ in the rearranged Equation 13. After, from the regular data set we calculate the mean of $C_{d}$, which is approximately independent of wave height and period. The second step is to simply use this mean $C_{d}$ value to derive $q_{C_{d}}$ using Equation 13. This GUA example demonstrates how $C_{d}$ can be derived using data based on assumed values before the experiment, which was necessary to derive $q_{C_{d}}$ and enable the comparison of Methods (a) and (b). We present the results of this pre-experiment orifice calibration after describing the MCM sensitivity analysis of Methods (a) and (b), as follows.

Two sets of MCM simulations were set up as per Figure 6, one set for Method (a) and one for Method (b). The first step was to input nominal values of the quantities used to derive $P$. To obtain such nominal values required developing a simple mathematical model, based on the scale of the 
experiment, selected to be 1:30 based on the wave basin characteristics (location, dimensions, water depth, and wavemaker capability) and other practical aspects including model build and instrumentation implementation. The model consisted of an assumed sinusoidal wave profile of 101 data points representing the free surface elevation inside the OWC, $\eta_{o w c}=A_{\eta_{o w c}} \sin (k x-\omega t)$ where $A_{\eta_{o w c}}$ is the amplitude. From this profile, $q$ was derived using Equation 12, assuming the cross-sectional area of the OWC to be $S_{c}=0.168 \mathrm{~m}^{2}$ (based on previous knowledge). In turn, we derived $p$ from $q$ by rearranging Equation 13, and substituting $C_{d}=0.6$ (the theoretical discharge coefficient), air density $\rho_{a}=1.2$ $\mathrm{kgm}^{-3}$, and orifice area $A_{0}=0.011 \mathrm{~m}^{2}$ (based on available knowledge). Finally, $P$ was calculated from $P=p q$. Figure 7 shows the results from this modelled OWC system, where all profiles are normalised against their maximums to conveniently graph them together. $p$ and $q$ are negative because the WSE WEC has a unidirectional flow PTO, described above in section 3 and elaborated on below.

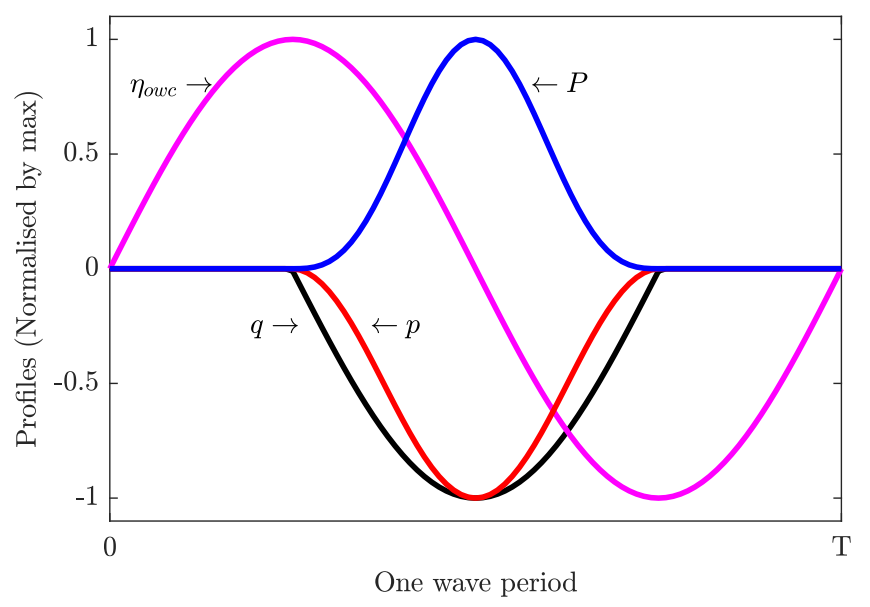

Figure 7: Profiles of variables, normalised against their respective maximums for one wave period

Nominal values obtained from above and visualised in the profiles of Figure 7 were then input into two sets of MCM simulations. The MCM was implemented here by subjecting the 101 data points of the profiles to the random sampling process. That is, at each iteration, every data point has an assigned uncertainty value which is multiplied by a randomly sampled number. After $M$ iterations, each data point is an M-by-1 vector that forms a PDF, such that the 101 PDFs for each data point form the uncertainty bounds along the profile. From the uncertainty bounds, the uncertainty was summarised at one point along the profiles - the amplitude. This will become clear once we present the relevant results below.

In Method (a), sixteen simulations were run consisting of combinations of input uncertainty values of pressure $u_{G}(p)$ and OWC internal free surface elevation $u_{G}\left(\eta_{o w c}\right)$, across a range of amplitudes of OWC internal free surface elevation $A_{\eta_{\text {owc }}}$. Figure 8 presents these MCM simulation results, which show $U_{G}(P)$ decreased as $A_{\eta_{\text {owc }}}$ increased. This result was due to the absolute uncertainties of input 
quantities $u_{G}(p)$ and $u_{G}\left(\eta_{\text {owc }}\right)$ being relatively smaller than the amplitudes of those quantities. Also, $U_{G}(P)$ was sensitive to a small change in $u_{G}\left(\eta_{\text {owc }}\right.$ ) (from 1 to $2 \mathrm{~mm}$ ), whereas an equal relative

three but preferably six wave probes installed in the OWC, so the $\eta_{\text {owc }}$ free surface behaviour is more accurately captured and averaged.

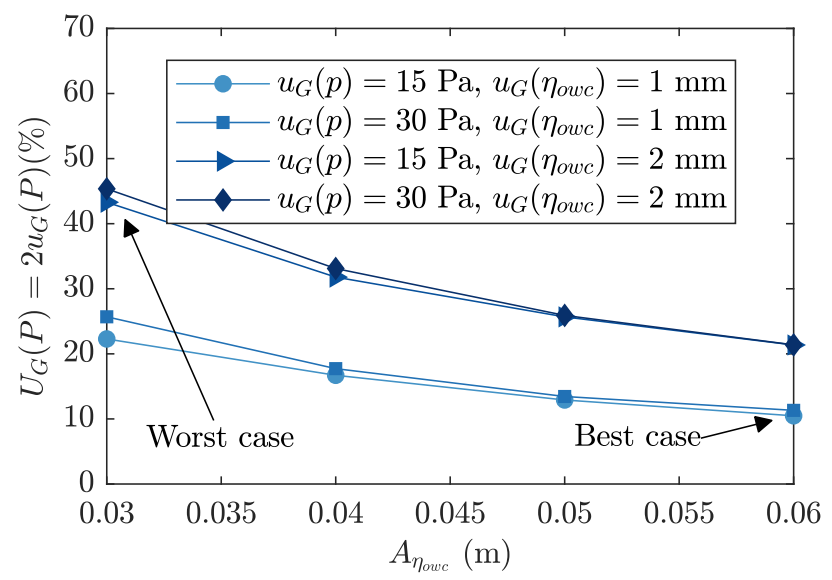

Figure 8: MCM simulation results of Method (a), showing the sensitivity of general expanded uncertainty $U_{G}(P)$ in OWC power $P$ to a combinatorial set of input uncertainty values of pressure $u_{G}(p)$ and OWC internal free surface elevation $u_{G}\left(\eta_{o w c}\right)$, across a range of amplitudes of OWC internal free surface elevation $A_{\eta_{o w c}}$.

In Method (b) a similar set of MCM simulations were run. To compare the MCM results between Methods (a) and (b), we show results in Figure 9 from the best case and worst case as highlighted in the Method (a) results (Figure 8). In Figure 9 the coloured bands on the profiles are the uncertainty distributions for each data point. Inset onto each subplot is a histogram showing the MCM produced PDF of $U_{G}(P)$ at the amplitude of $P$. The titles of the subplots specify the assumed nominal and uncertainty values for each case. In the Method (b) MCM results there is an additional uncertainty value assumed for volume flow rate $u_{G}\left(q_{C_{d}}\right)$, which is of approximate equal magnitude to the quantities' uncertainties seen in Method (a) MCM results.

$U_{G}(P)$ was about twice as small in Method (b) MCM results compared to Method (a), for both the best and worst cases (Figure 9). An explanation of this result is the following. $C_{d}$ used in Method (b) is averaged from all the pressure-flow characteristics in the OWC under all wave conditions, and 


\section{Method (a) MCM results}
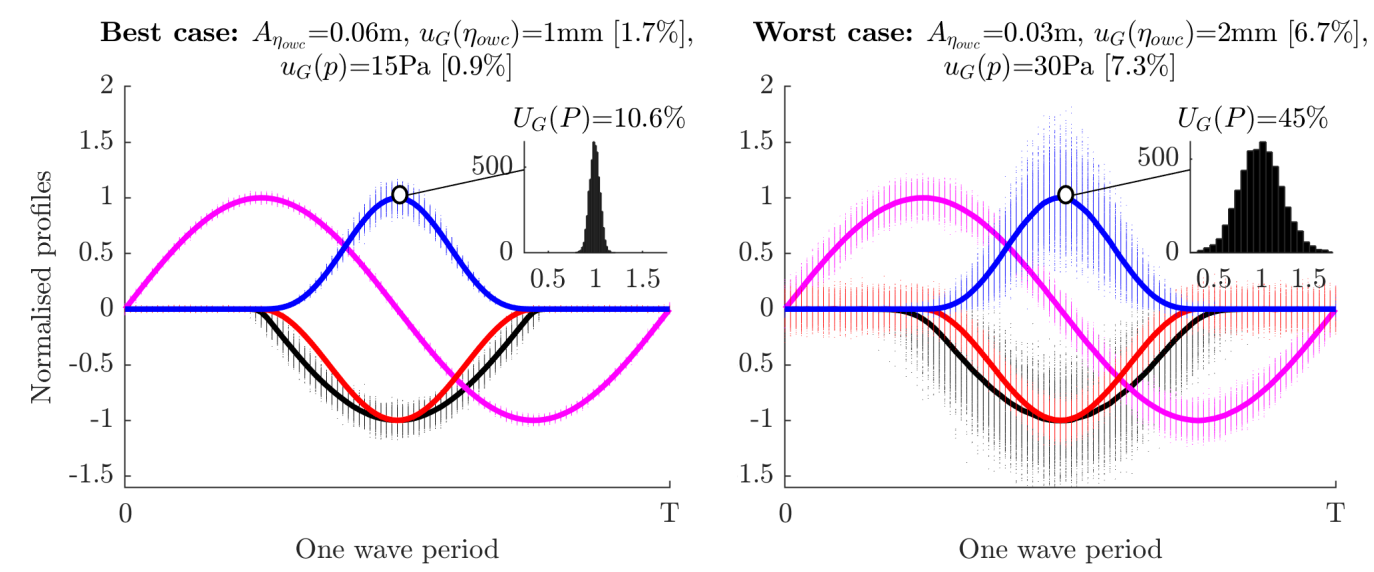

\section{Method (b) MCM results}
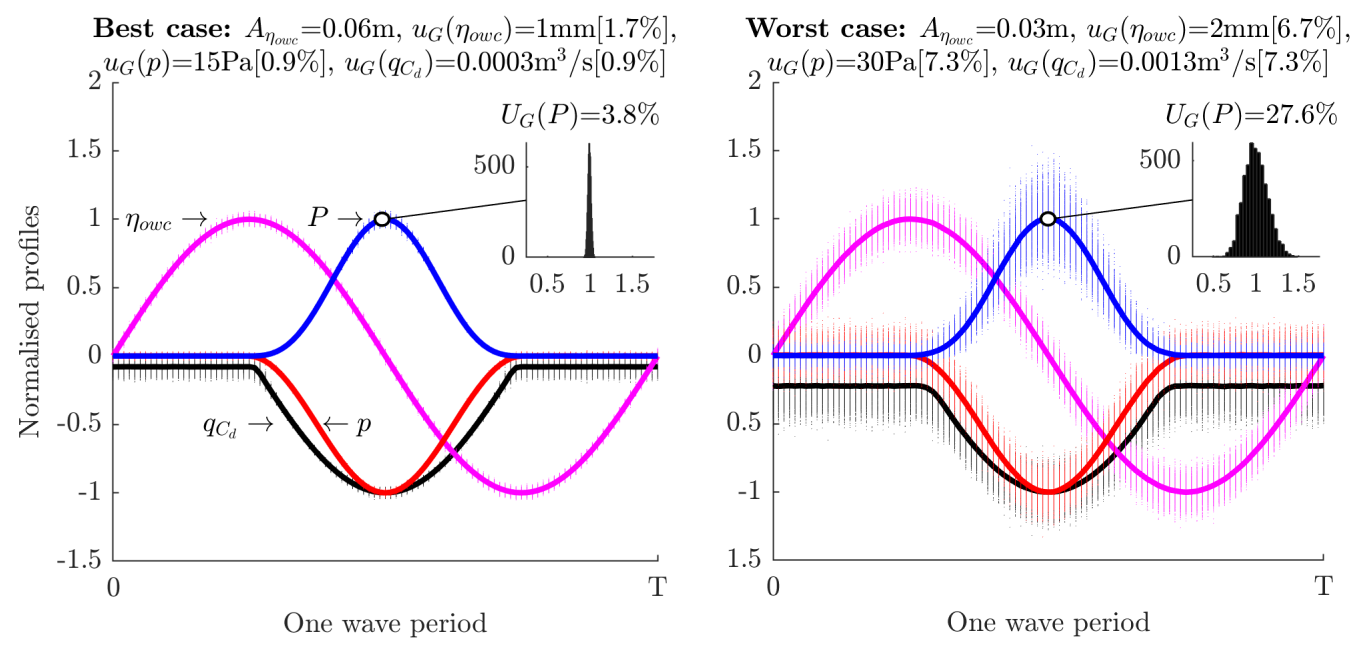

Figure 9: MCM simulation comparison results, showing for each method the best case (smallest relative uncertainty) and worst case (largest relative uncertainty). Graph titles specify nominal and uncertainty values (uncertainty values are \pm ). Histograms show normalised $P_{\max }$, where $U_{G}(P)=2 u_{c}(P) . U(P)$ in Method (a) MCM results was higher than in Method (b) MCM results, therefore, Method (b) is better.

it is approximately independent of wave height and period. Such averaging reduces the uncertainty in $q_{C_{d}}$ (therefore $P$ ). Conversely, in Method (a), $q_{\eta_{o w c}}$ is sensitive to the nonlinear sloshing behaviour of the $\eta_{\text {owc }}$ free surface elevation, which depends on wave height and period. Based on these MCM results, Method (b) clearly produces the least uncertainty and was therefore chosen to derive $P$ in the experiment.

The general uncertainty value of the orifice discharge coefficient $u_{G}\left(C_{d}\right)$ used in the above MCM sensitivity analysis for Method (b) was obtained from the pre-experiment orifice calibration procedure. Figure 10 shows the results of this calibration, where $C_{d}$ is plotted against $p$. The $C_{d}$ data in this figure were generated through a MCM simulation, using the same quantity profiles and uncertainty 
values assumed for the main MCM sensitivity analysis described above. These data are representative of all the regular wave data of the intended experiment. The black data points bounded by red dashed lines (two standard deviations $\sigma$ ) and $p<200 \mathrm{~Pa}$ were included in the calculation of the mean of $C_{d}$, and its standard deviation equal to the general uncertainty $u_{G}\left(C_{d}\right)$.

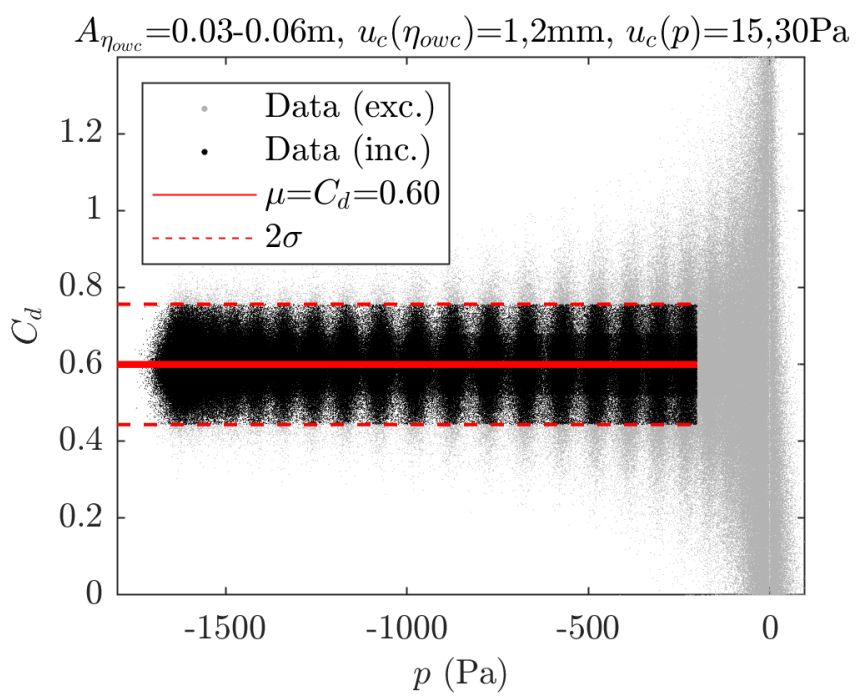

Figure 10: Orifice calibration graph showing the $C_{d}-p$ relationship, and the method to estimate an appropriate value for $C_{d}$ within defined limits.

The foregoing example demonstrates but one of the many uses of GUA and the MCM to help plan an experiment and design some aspects of it. It is exemplary of the process that can be undertaken when a new procedure is introduced in an experimental investigation. The technical outcomes and information generated in this GUA are (1) an informed decision on the least uncertainty method to derive OWC power, and (2) an enhanced understanding of OWC power sensitivity to a range of nominal and uncertainty values of its input quantities. Moreover, the knowledge gained in (2) revealed critical measurements to be made - such as the OWC internal free surface using an appropriate number of wave probes - and where extra attention may be required to assure the desired uncertainty level is achieved.

More broadly, this section argues for the value of GUA, that it is worthwhile because it generates otherwise inaccessible insights and information which can inform the subsequent design phase and create the conditions for a successful, high-quality experiment. It follows that new procedures developed in a laboratory should be linked to a GUA and, once applied, to a DUA as part of quality control. If similar experiments using well developed procedures are performed, the previously developed GUA could be used. Because WEC testing often brings new complexity (e.g. valves, PTO control, targeted wave climate), however, a new GUA may often need to be developed. Even if a seemingly similar ex- 
periment is performed, some small changes in parameters (wave properties, different instrumentation etc.) could change the results of the GUA. Checking that the parameters fit within the boundary of tion 4.1). The only difference is the measurands $y$ and input quantities $x_{i}$ may now be considered as an expectation (the result of a measurement), so the notation is lower case: $y=f\left(x_{1}, x_{2}, \ldots, x_{N}\right)$.

\subsection{Stage 1b. Formulate: Identify uncertainty sources} uncertainties of the $x_{i}$ are considered in more detail. Figure 11 shows an example of a cause-and-effect diagram used to identify uncertainty sources for $P_{W}$ and $P$.

\subsection{Stage 1c. Formulate: Evaluate standard uncertainty}

Every $x_{i}$ has a standard uncertainty associated with it, consisting of components evaluated using two methods: Type A and Type B. Evaluating Type A standard uncertainty $u_{A}\left(x_{i}\right)$ requires estimating the mean $\bar{q}$ of $n$ independent observations/repeats $q_{k}$ which are characterised by a PDF,

$$
\bar{q}=\frac{1}{n} \sum_{k=1}^{n} q_{k},
$$




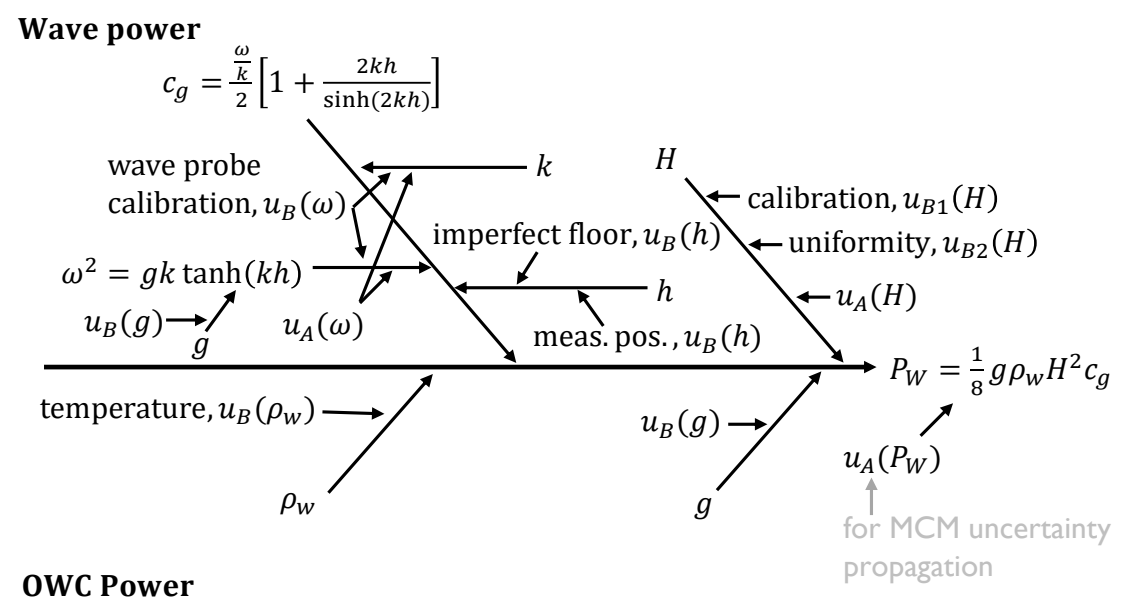

OWC Power

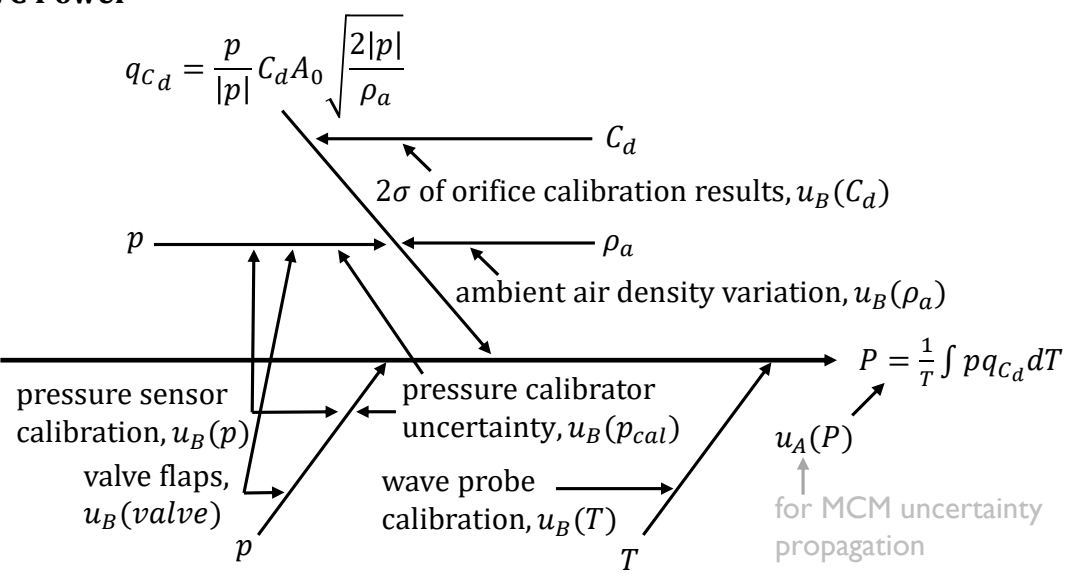

Figure 11: A cause-and-effect diagram used to identify uncertainty sources. Example is of wave power $P_{W}$ and OWC power $P$, where the $u_{A}\left(x_{i}\right)$ is the Type A uncertainty and $u_{B}\left(x_{i}\right)$ the Type B uncertainty.

and the experimental standard deviation of the mean $s(\bar{q})$,

$$
u_{A}\left(x_{i}\right)=s(\bar{q})=\sqrt{\frac{\frac{1}{n-1} \sum_{j=1}^{n}\left(q_{j}-\bar{q}\right)^{2}}{n}} .
$$

The general conditions for repeatability are [31, 28]: the same measurement procedure; the same measuring instrument used under the same test conditions; the same laboratory or field site; and repetition over a short period of time, roughly one day. The repeats should comprise sequential and non-sequential runs.

Evaluating Type B standard uncertainty $u_{B}\left(x_{i}\right)$ requires judgement, experience, and all available information to first identify which of the large number of possible uncertainty sources are significant and, second, to estimate numerical values for the significant sources. Means other than statistical analysis are used, but $u_{B}\left(x_{i}\right)$ is also characterised by the standard deviation of an assumed PDF. 
Assigning the $u_{B}\left(x_{i}\right)$ PDF, or a set of PDFs as often quantities have several Type B sources, is based on the scientific judgement of a pool of comparatively reliable information on the possible variability of $x_{i}$ [31]. As the experimental program progresses so to increases the available $u_{B}\left(x_{i}\right)$ information. At the design phase, the information typically consists of previous measurement data, previous experience with or general knowledge of the phenomena or process, reference material provided by suppliers in terms instrument calibrations and other certificates, and uncertainties assigned to reference data taken from handbooks. In subsequent experimental phases, the available information increases as apparatus is assembled, instrumentation installed, calibrations performed, and experimental runs performed [30].

\subsection{Stage 2. Propagate: Determine combined uncertainty}

To determine the combined standard uncertainty $u_{c}(y)$, identified standard uncertainties of $x_{i}$ 's are propagated through the measurand function $y$. Figure 12 shows the general process of MCM uncertainty propagation in DUA, for any number of measurand levels. First, we input measured nominal values of each quantity $x_{i, n o m}$, and $u_{B, k}\left(x_{i}\right)$. The $u_{A}(y)$ is also input for the top-level measurand $y$ (in this example, $u_{A}(y)$ is included only for the top-level measurand, however, $u_{A}\left(x_{i}\right)$ can be used for each $x_{i}$ and propagated that way - see [30]). The standard uncertainty components are assumed to be the standard deviations of their PDFs. The PDFs for $u_{B, i}\left(x_{i}\right)$ are here assumed to be Gaussian, but others may be used. If two $x_{i}$ 's share a $u_{B}$, or two $x_{i}$ 's are correlated, a joint PDF can be assigned. Then, at each iteration $\mathrm{j}, u_{B, k}\left(x_{i}\right)$ is multiplied by a randomly sampled number from the assumed PDF (varying about 1 ), and added to $x_{i, n o m}$ obtain the 'measured' values $x_{i}(\mathrm{j})$. The top-level measurand $y(\mathrm{j})$ is then calculated with the included $u_{A}(y)$ term multiplied by a randomly sampled number from the PDF, comprised of the repeated observations of $y$ in the experiment. The sampling process is repeated $M$ times to obtain a PDF for $y$. The output of the MCM is the standard deviation of the PDF of $y$, taken as the combined standard uncertainty $u_{c}(y)$.

\subsection{Stage 3. Summarise: Summarise uncertainties}

As in GUA, we use the PDF of $y$ to obtain: the mean and standard deviation of $y\left(u_{c}(y)\right)$, and a coverage interval $k$, giving the expanded uncertainty $U=k u_{c}(y)$. This summary stage includes presenting the detailed uncertainties in key quantities and measurands and any other important uncertainty-related information. The presentation generally consists of uncertainty results presented in tables or graphs, in the form $x_{i} \pm u_{A, B}\left(x_{i}\right)$ and $y \pm U$.

The remainder of this section describes the details of the OWC WEC experiment and demonstrates how DUA was and could be used at key experimental phases. 


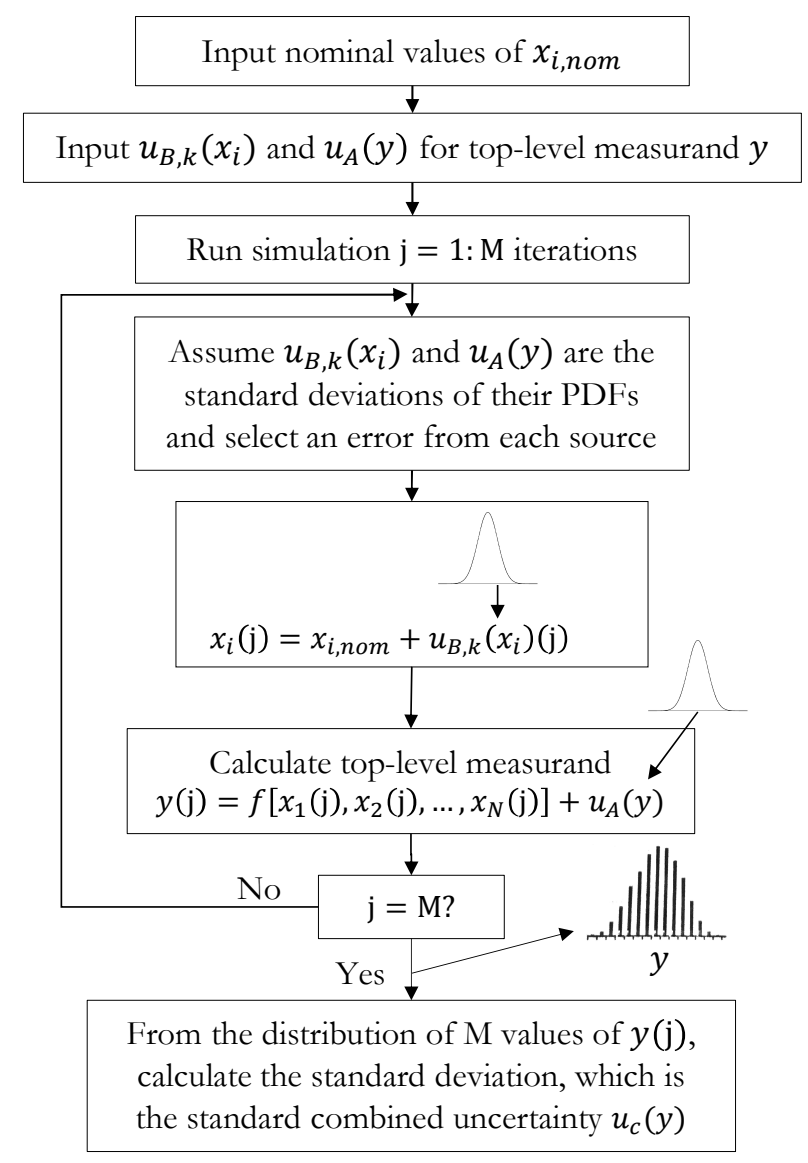

Figure 12: Flow diagram of the Monte Carlo Method to propagate uncertainty in detailed uncertainty analysis. The example shows the flow of the MCM when there are multiple levels of measurands with one or more input quantities.

\subsection{Design, construct, and debug}

DUA can inform the design, construction, and debugging phases of an experiment. The design phase builds on GUA-generated information to specify instrumentation and details of experimental apparatus. The test plan, parameters, and procedures are identified and decisions made on the data to be obtained, and the scope and sequence of conditions and runs. This process can be guided by Design of Experiment [28. Technical drawings of the model are also issued for fabrication.

The parameters of this experiment are summarised in Figure 13 and Table 2.

In the construction phase the apparatus is assembled, calibrations carried out, and initial runs performed. The debugging phase follows, in which unforeseen problems are addressed. The completion of these phases is indicated by the apparatus operating as expected and factors influencing uncertainty in the results well understood. In addition, incoming data are monitored using built in checks to guard against unnoticed and unwanted changes in the apparatus or operating conditions [30. DUA can inform all these processes. The following subsections, however, focus on how DUA was applied to 
Model mounted on force balance
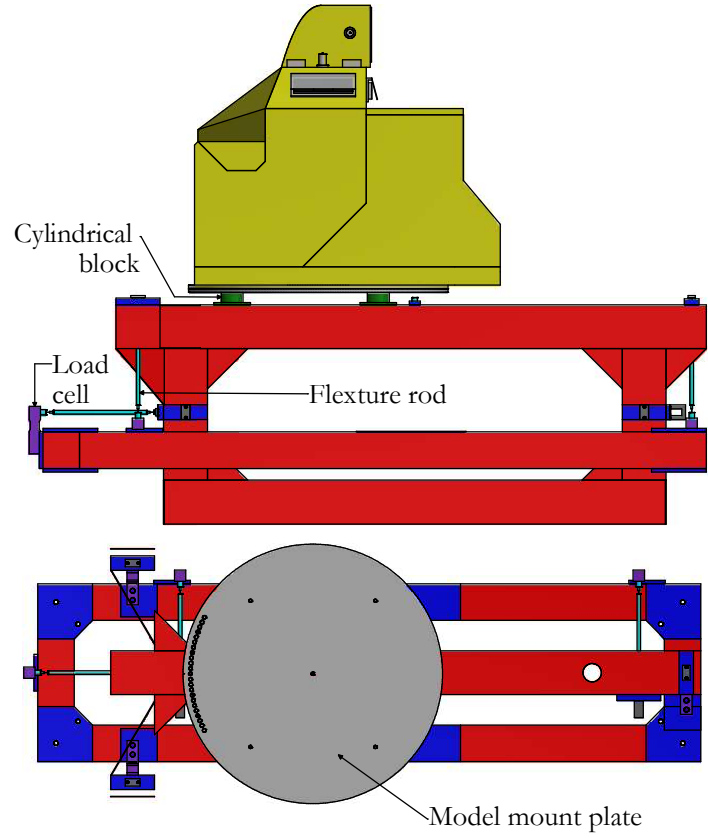

Instruments installed in OWC
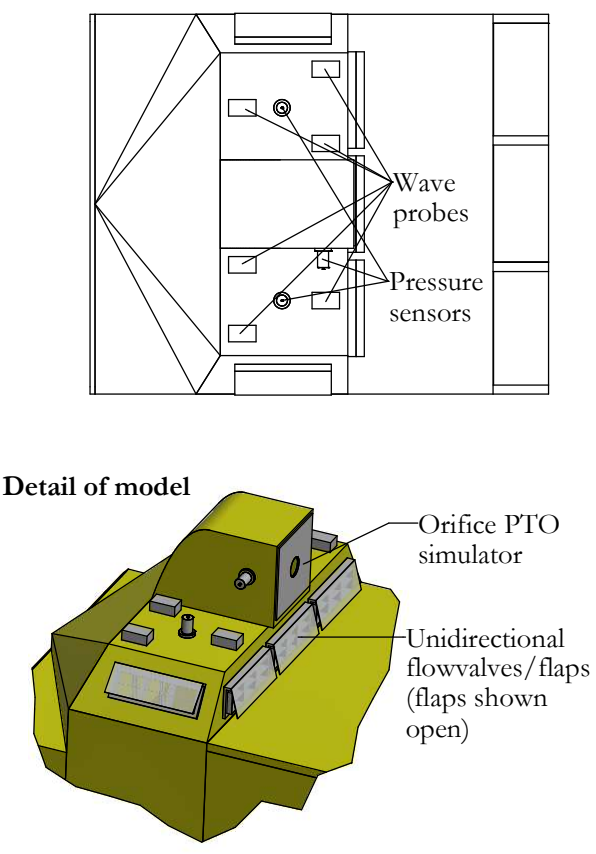

AMC wave basin

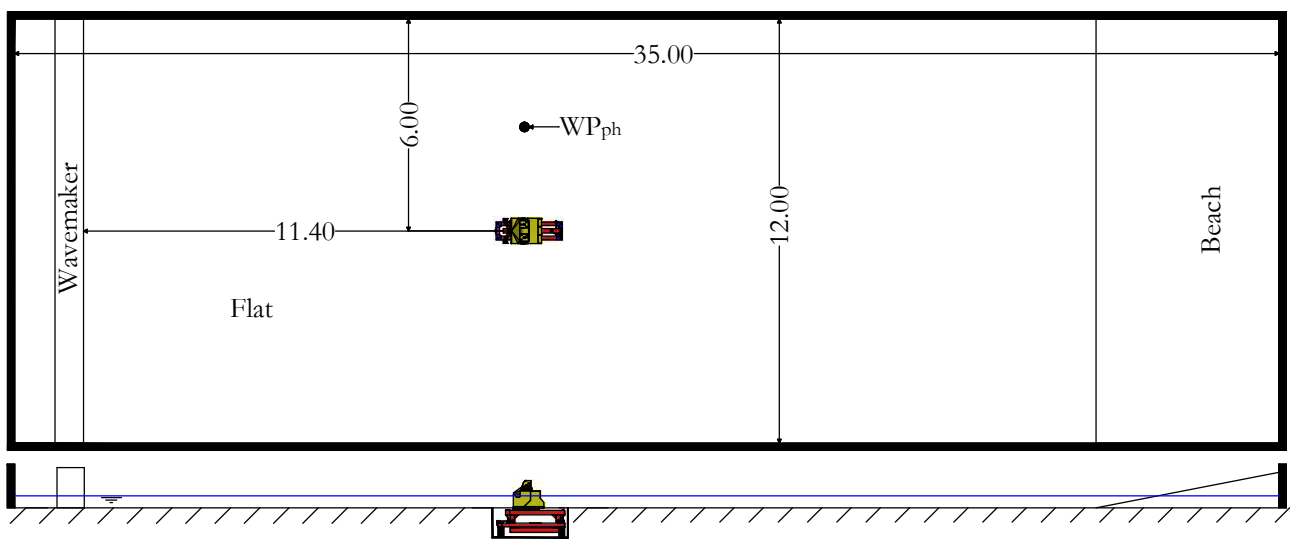

Figure 13: Diagram of important aspects of the OWC WEC model and the AMC Model Test Basin. Annotations reveal instrumentation configurations and the general layout of the model installed in the wave basin. $W P_{p h}$ is the phase wave probe.

assure and quantify the quality of instrument calibrations in the construct phase and throughout the experiment.

\subsubsection{Example: Evaluating Type B uncertainty from instrument calibrations}

For wave probe $(\mathrm{WP})$ calibrations, to evaluate $u_{B}(W P)$ involved calculating standard error of the estimate (SEE) of the WP calibration curve: 
Table 2: Parameters of the experiment. Full-scale values given. Apply the Froude scaling law to obtain model-scale values

Parameter

Model

Environment

Measurements;

instrumentation;

calibrations

Data acquisition

Data recording

Wave conditions

Model conditions

\section{Details}

Bottom-standing unidrectional flow OWC WEC; 1:30 Froude-scale model $\left(\lambda_{30}\right)$; body constructed from marine plywood, Perspex, and fibreglass; orifice plate PTO simulator (1:150 orifice/chamber area ratio); valves simulated with passive flaps (thin, robust plastic sheets), but mass properties not scaled as prototype material was unknown at the time.

Shallow water wave basin (35m L x 12m W x 0-1m D); 16-element piston-type wavemeker; vertical walls; passive beach; fresh water (15-20 C); water depth for experiments $=10 \mathrm{~m}$.

Wave elevation in basin, conductive wave probe, calibrated daily; wave elevation in OWC, 6 conductive wave probe (see Figure 13 for layout), calibrated daily; pressure in OWC, 3 x Honeywell Controls TruStability pressure sensor connected to Ocean Controls KTA-284 instrumentation amplifiers, calibrated weekly to $\pm 2000 \mathrm{~Pa}$; hydrodynamic loads on model, 6-component force balance, calibrated before and after each scale experiment.

National Instruments PCI-6254M Multifunction Data Acquisition Card, recorded on a HP computer, controlled with Labview software. All data acquired at $200 \mathrm{~Hz}$.

Regular waves: 30 seconds (model-scale); irregular waves: 30 minutes.

Regular waves: $H=1.8,2.4,3.0 \mathrm{~m}, T=8-15.8 \mathrm{~s}(k h=0.41-0.98)$; irregular waves (JONSWAP): 15 sea states: $H_{s}=0.75-4.75 \mathrm{~m}, T_{p}=7-19 \mathrm{~s}$.

Incident waves without model: all waves; power matrix: operational waves; loads: operational waves.

$$
u_{s, B}=S E E=\sqrt{\frac{\sum\left(y_{j}-\widehat{y}_{j}\right)^{2}}{M-2}}
$$

415

where $M$ is the number of calibration points, $y_{j}$ is the calibrate data point, and $\hat{y}_{j}$ is the fitted value.

Figure 14 shows an example of a wave probe calibration curve, where $u_{B}(W P)=S E E$. The residuals (blue squares) emphasise the variation of each data point (black circles) about the regression line. The $u_{B}(W P)$ estimate here forms one component of the multiple $u_{B}\left(x_{i}\right)$ components of $\eta_{i n c}$ and its related wave parameter quantities of $H, T, c_{g}$, etc. (see Figure 5). A similar process can be carried out to estimate $u_{B}\left(x_{i}\right)$ for other instruments such as pressure transducers and load cells. For 
a pressure transducer, however, there is an additional significant $u_{B}$ component to estimate, which is the pressure calibrator used to calibrate the pressure transducer in the first place. Such an estimate is based on manufacturers specifications.

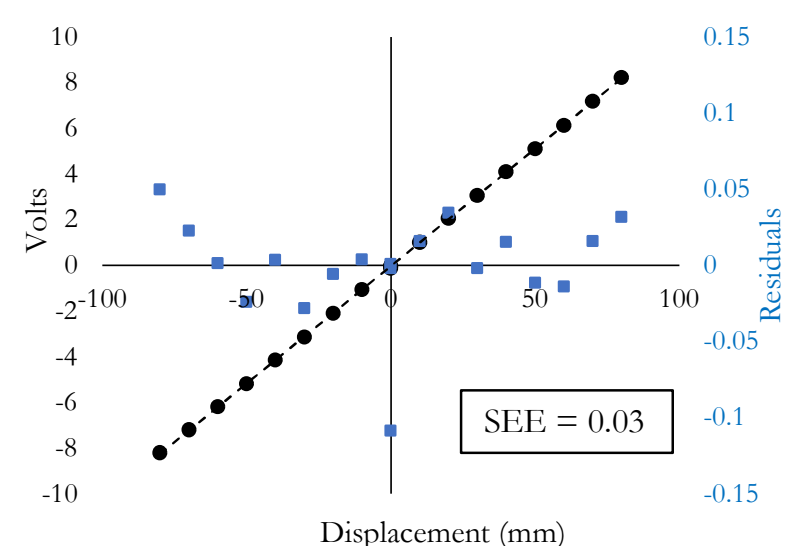

Figure 14: Example of a calibration curve of a wave probe, with included residuals and SEE calculation.

\subsection{Execute}

In this phase, in which experimental data are collected, the focus shifts to evaluating $u_{A}(y)$ based on statistical analysis of repeats. A top-down approach may be applied to generate a data set from 
which to calculate $u_{A}(y)$ of the top-level $y$. If this $u_{A}(y)$ data set cannot be produced directly, for instance if $y$ is a function of lower-level measurands/quantities $x_{i}$ that are measured/derived in different experimental conditions, then the $x_{i}$ upon which $y$ depends can be split up and data sets produced for the groups of $x_{i}$. This top-down approach is illustrated in the following examples for regular and irregular waves, respectively.

\subsubsection{Examples: Evaluating Type A uncertainty}

The top-level power measurand is $C_{W}$ (Equation 11), which is a function of $P$ (Equation 10), $P_{W_{r}}$ Equation 1), and $B$. It was not possible to produce one data set of repeats to calculate $u_{A}\left(C_{W}\right)$ directly, because $P_{W_{r}}$ required measurements of incident waves at the WEC model location without it installed, and $P$ required the model to be installed. So, there needed to be two separate $u_{A}\left(C_{W}\right)$ data sets, one for wave power $u_{A}\left(P_{W_{r}}\right)$ and one for OWC power $u_{A}(P)$.

Regarding $u_{A}\left(P_{W_{r}}\right)$, a reasonable data set consisted of at least ten repeats $n$ 28] of regular waves at several set points and experimental conditions, in this case, several wave periods and wave heights spanning their respective ranges. An $n$ was considered to be not a regular wave run, but rather an individual wave within runs, as per the method developed in [22]. (This method effectively reduces the number of required runs from ten runs to 3-5 runs, thereby saving time and money.) The $x_{i}$ that significantly contribute to the random variation in $P_{W_{r}}$ are $H$ and $c_{g}$, both of which are derived from $\eta_{\text {inc }}$. Thus, the $u_{A}\left(P_{W_{r}}\right)$ data set was produced by 3-5 repeat runs of incident waves at the model location without it installed. Each set of repeats consisted of a low, medium, and high wave period, at multiple wave heights. From this data set, $u_{A}\left(P_{W_{r}}\right)$ could be calculated.

The $u_{A}(P)$ data set was similarly obtained, with the same set of repeat runs, except with the model installed. A similar $u_{A}(y)$ evaluation process was carried out for the $F x, F z$, and $M y$ measurands.

Figure 15 shows a visualisation of key $u_{A}(y)$ data sets, for one regular wave height. The profiles show (1) individual waves representing independent repeats $n$; (2) the several representative set points of repeats, here with three set points of the lowest, mid, and highest wave period; and (3) how the variation in the key input quantity $\eta_{i n c}$ induces a similar variation in all other dependent quantities of $\eta_{o w c}, p, P, F x, F z$, and $M y$. Depending on the quantity, the magnitude that is used to calculate $u_{A}(y)$ is either an amplitude (as for $F x, F z$, and $M y$ ), a height (as for $\eta_{i n c}$ ), or an integral (as for $p$ and $P)$. Noted, $u_{A}(y)$ or $u_{A}\left(x_{i}\right)$ should be calculated whenever more information becomes available during the experiment. This simple, useful practice allows us to track uncertainties and decide if more repeats are needed to drive down the uncertainty in the results. All $u_{A}(y)$ results are summarised in Figure 18,

Evaluating $u_{A}(y)$ in irregular waves was similar to regular waves. The process involved performing at least five repeats for five sea states that ranged the tested wave climate (five repeats only due to 

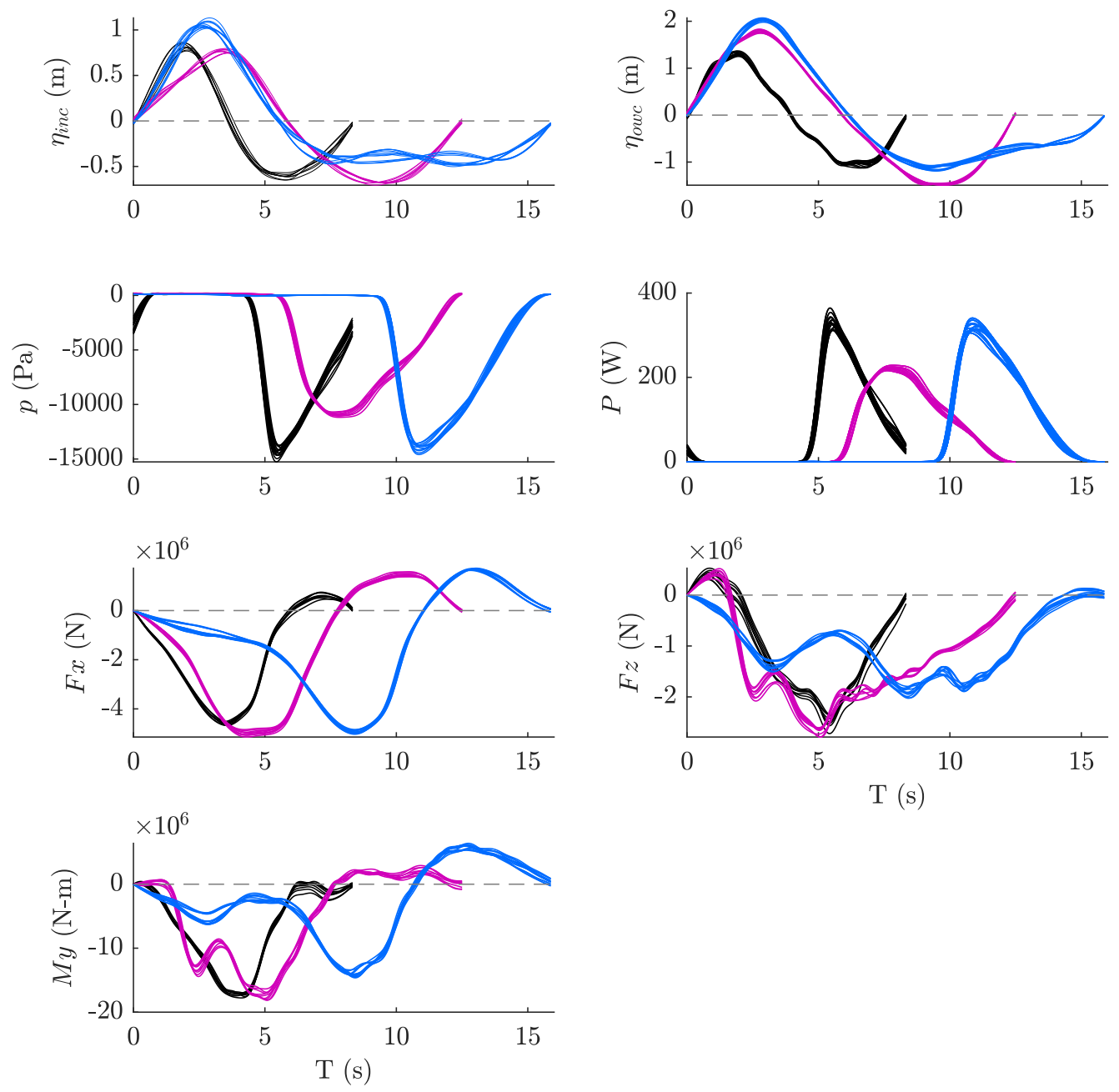

Figure 15: Overlayed wave profiles of individual waves taken from time series data of repeated independent observations. Plots show three wave periods (lowest, mid, highest) and one wave height. The magnitudes (e.g.., the amplitude, height, or integral) calculated from each individual wave are input into the Type A uncertainty calculation.

time constraints: irregular wave runs are much longer than regular wave runs). From these data sets the power-related and load statistics were calculated, and an average taken to ascribe one value for the entire power matrix and load matrix. These $u_{A}\left(x_{i}\right)$ values were then used as input, along with the $u_{B}\left(x_{i}\right)$, into the Monte Carlo simulations to propagate uncertainty to determine combined uncertainty $u_{c}(y)$. The next section describes this process and presents its results.

\subsection{Analyse and report}

Here we first describe the data analysis techniques used in the experiment, then describe and give examples of DUA applied in these experimental phases. Data were analysed according to the ITTC Recommended Procedures and Guidelines [49. For the regular waves analysis, timeseries data were 
trimmed to include only the 'stationary' region, omitting transitional and reflected waves, thereby avoiding having to perform a reflection analysis (see [50] Fig. 9). A phase-averaging technique was applied to the trimmed timeseries to reduce the repeating wave cycles into one representative, 'phaseaveraged' wave cycle 21. This technique effectively reduces uncertainty and improves results presentations. For the irregular waves analysis, a Fast Fourier Transform (FFT) was applied to the cropped timeseries data. Welch's power spectral density estimate method (pwelch function in MATLAB) was used to transform time domain data into the frequency domain. Irregular wave parameters (significant wave height, energy period, etc.) were calculated from the spectral moments of the energy density spectrum as per subsection 4.1

\subsubsection{Example: Determining combined uncertainty}

$u_{c}(y)$ was determined by propagating uncertainty using the Monte Carlo method. For regular waves, Monte Carlo simulations were set up as per Figure 12, with one simulation for each regular wave period and height. The assigned $u_{A}\left(x_{i}\right)$ and $u_{B}\left(x_{i}\right)$ was unique for each wave period and height, differing depending on the number of repeats, the instrument calibration corresponding to the data, and other $u_{B}\left(x_{i}\right)$ sources. The power-related and load measurands were calculated for $M=10,000$ iterations, from which $u_{c}(y)$ was calculated. A 95\% coverage interval was applied to the PDFs of the $y$ 's to obtain the expanded uncertainty $U$. A similar process was performed for irregular wave results. The main differences were (1) one MCM simulation per sea state, and (2) for each sea state the $u_{A}\left(x_{i}\right)$ was a unique, averaged value of the repeats from five sea states (as described in the above example). The results from the data and uncertainty analyses are shown in Figure 16 for regular waves and Figure 17 for irregular waves. Figure 18 summarises the uncertainties in key measurands in a table-like format.

\subsubsection{Example: Summarising uncertainties}

Regular wave results showed a reasonable level of uncertainty (Figure 16 and Figure 18). The largest expanded uncertainty overall was in $C_{W}$, averaging $\pm 16 \%$ with a maximum of $\pm 25 \%$. These uncertainty levels were expected considering $C_{W}$ is a top-level measurand that is a function of $P_{W}$ and $P$, which are themselves functions of many inputs, thereby making $C_{W}$ sensitive to uncertainty due to its many inputs. These uncertainty results are comparable to similar experiments [21, 22] and to similar work [19, 20, 51]. The uncertainty in loads of $F_{x}, F_{z}$ and $M_{y}$ were relatively smaller, averaging $\pm 6 \%$ with a maximum of $\pm 10 \%$. The uncertainty in $k h$ was small, less than $\pm 2 \%$, which is to be expected for wavemakers that generate highly repeatable wave period. In both regular and irregular waves, $u_{B}$ tended to be larger than $u_{A}$.

Uncertainty results in irregular waves are summarised in a power matrix and load matrix (Figure 17) and in Figure 18 (see figure caption for guidance on reading the plots in Figure 17). The uncertainty 

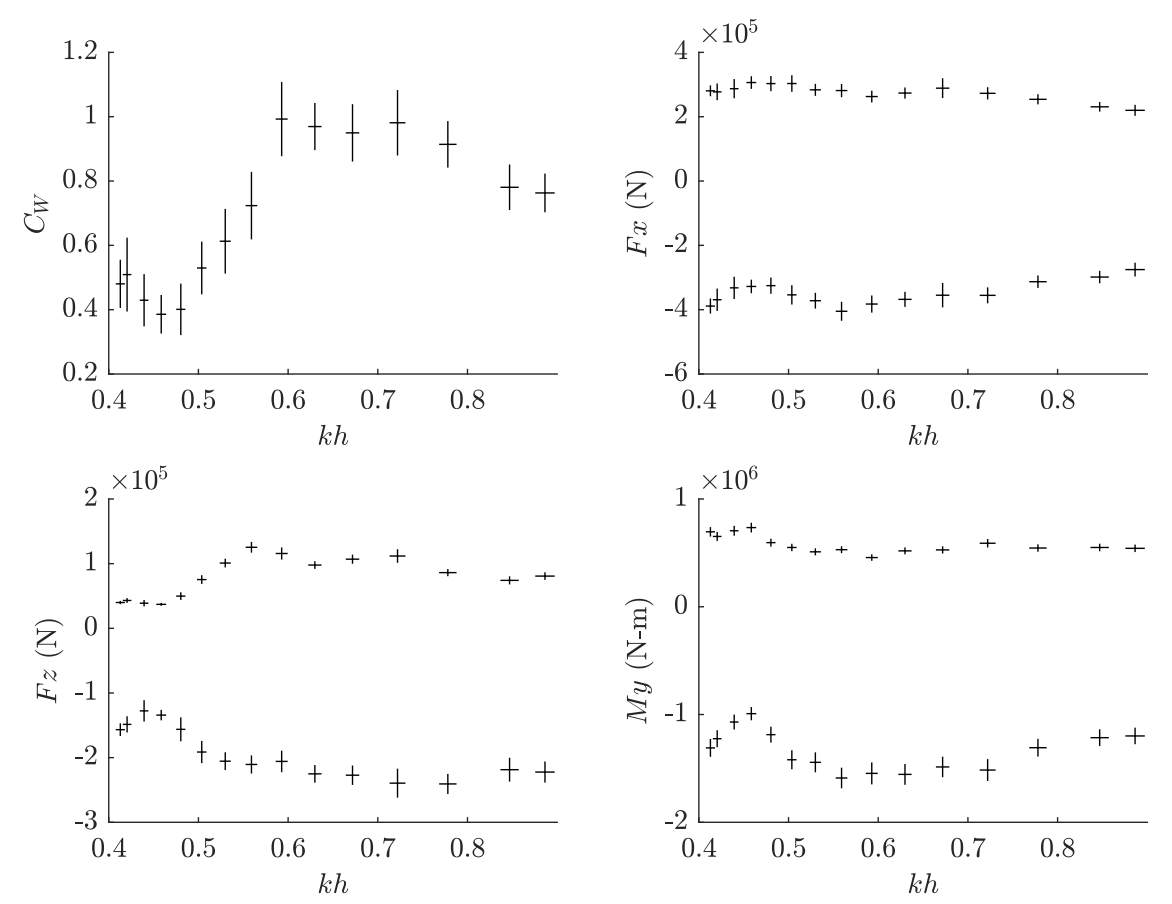

Figure 16: Key power and load measurands in regular waves, with error bars representing expanded uncertainty to $95 \%$ coverage interval.

results were similar to those in regular waves, but with $C_{W}$ uncertainty relatively smaller, averaging $\pm 11 \%$. The loads uncertainties were practically the same.

Key causes of uncertainty in $C_{W}$ were measurements used to derive the lower level measurands (wave power $P_{W}$ and OWC power $P$ ) and PTO modelling. For $P_{W}$, the critical measurement, therefore key uncertainty in this measurand, was incident wave elevation. For $P$, the dominant uncertainties were measurements of $p$ and $\eta_{O W C}$ used to derive $q$ and, in turn, $C_{d}$. Another possible key uncertainty source in $P$ is the modelling of the valve flaps as robust yet lightweight flaps, whose mass properties were not froude-scaled to 1:30 scale. It is plausible that this aspect of the PTO modelling may be a dominant uncertainty in power, if in the prototype the valve flaps have a strong influence on OWC chamber dynamics. With neither knowledge of the mass properties of the prototype valve flaps nor full-scale results to compare with, it was difficult to assign a Type B uncertainty to the modelled valve flaps. This represents a modelling challenge that requires further research. However, because $u_{A}(P)$ was relatively small, this indicates that the simulated valve system was well-designed for the model-scale tests. 

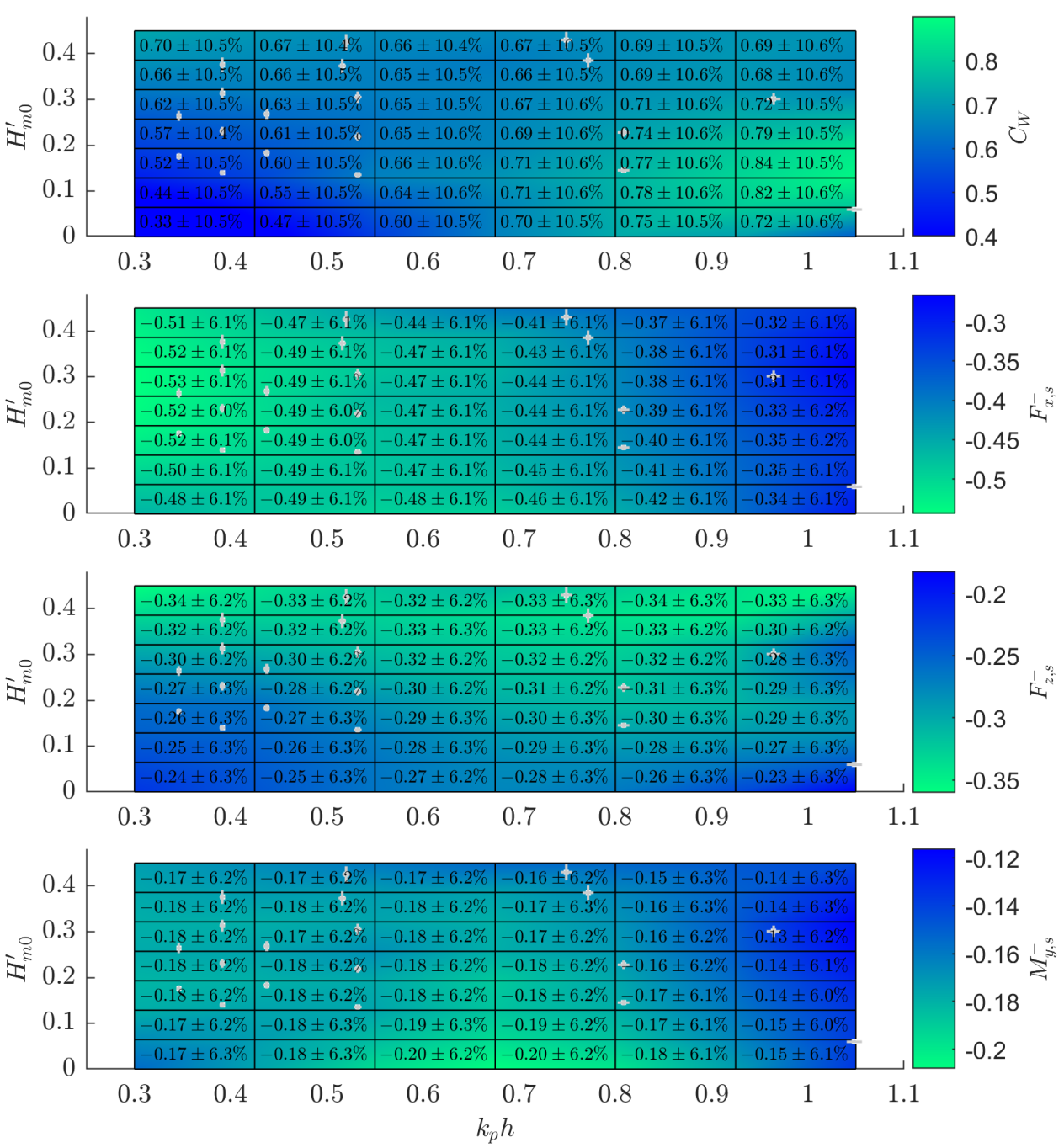

Figure 17: Key power and load measurands in irregular waves, for a matrix of $H_{m 0}$ vs $k_{p} h$ sea states. Measurands are dimensionless and normalised by the calculated $H_{m 0}$ for each sea state. The text in each coloured cell is in the form of "interpolated value of the measurand (z-axis) \pm expanded uncertainty. The white markers indicate the actual $H_{m 0} / T_{e}$ values, with error bars representing expanded uncertainty to $95 \%$ coverage interval. Colour of cells: green represents greatest magnitude, whether positive or negative.

\section{Discussion}

The foregoing sections present a general, comprehensive UA methodology applied to a WEC model test. The methodology features: a three-stage structure composed of the UA principles, applicable for both GUA and DUA; the use of cause-and-effect diagrams to identify uncertainty sources; the use of the MCM to propagate uncertainty; an approach to evaluate Type A uncertainty and a specific method for regular waves that reduces the number of required repeats, thereby saving time and money; 

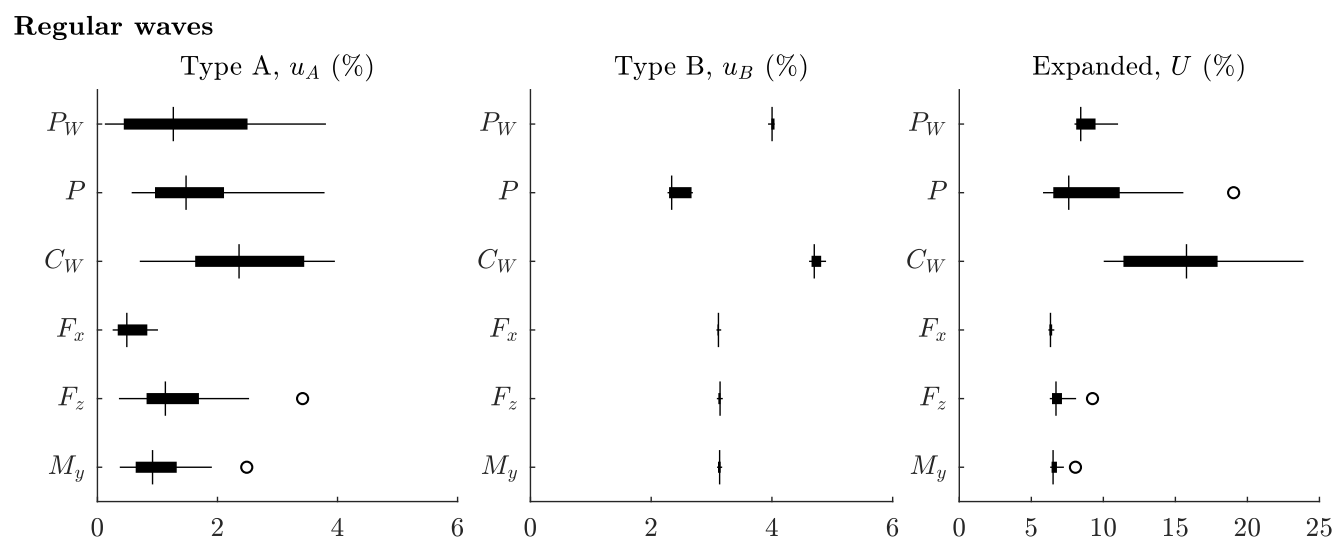

Irregular waves

Type A, $u_{A}(\%)$

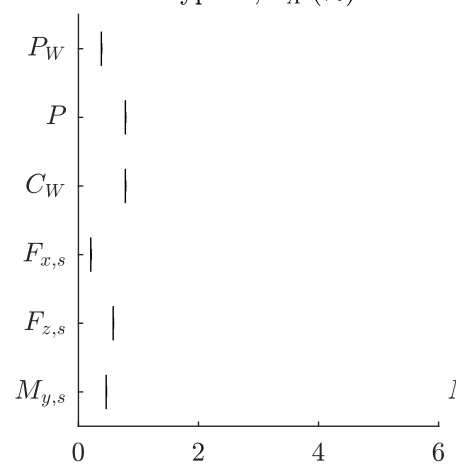

Type B, $u_{B}(\%)$
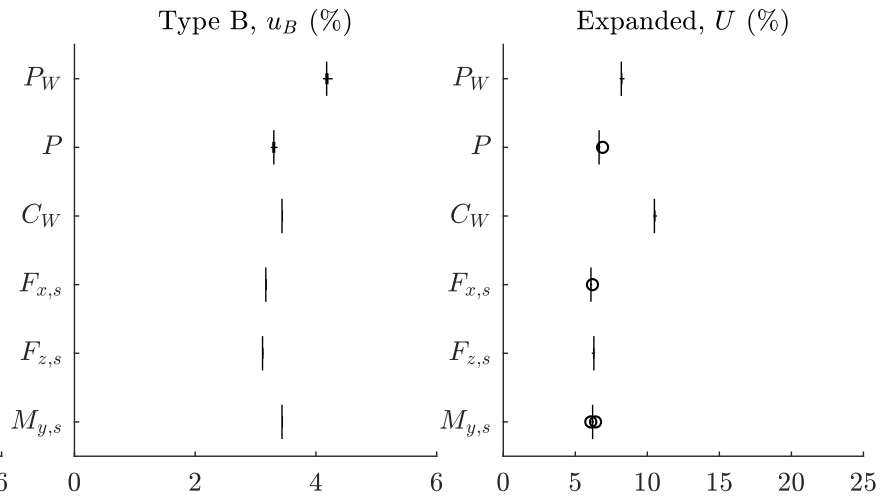

Figure 18: Uncertainty of key measurands shown as box and whisker plots (distributions). For Regular waves, the $u_{A}$ distribution includes all wave frequencies and heights; the $u_{B}$ distribution includes all instrument calibrations carried out throughout the experiment and other $u_{B}$ sources; the $U$ distribution includes all standard uncertainties, determined by the Monte Carlo Method. For Irregular waves, $u_{A}$ is based on five representative sea states, and $u_{B}$ and $U$ were obtained similarly as regular waves.

descriptions and examples of evaluating Type B uncertainty; and a method for UA in irregular waves. The methodology thus substantially extends current international guidelines on uncertainty analysis for WECs 28.

Findings in Figure 14 Figure 18 reveal several implications. The moderate uncertainty in $C_{W}$ suggests that, when extrapolating these results, uncertainty in predictions of prototype power performance is considerable. This uncertainty could lead to an ill-designed PTO system or inaccurate MAEP or LCOE predictions. On the wave power side, the use of linear wave theory to calculate wave power of the nonlinear waves may have introduced a non-negligible uncertainty into wave power calculations. This uncertainty could perhaps be reduced by applying an appropriate higher order theory [52, which may be investigated in future work. Regarding loads, though their uncertainty was relatively smaller, extrapolating still equates to an uncertainty of hundreds of tonnes. This uncertainty could lead to 
ill-defined design loads resulting in an under- or over-designed main structure or foundations, thereby impacting CapEx and OpEx. Summarising, when extrapolating results it is important to consider how the uncertainty may impact how the results are used; if the uncertainty is deemed too large in a measurand, it may need to be reconsidered, either by further experiments or a more rigorous uncertainty evaluation.

Regarding $C_{W}$, as it is a function of many inputs it is inherently sensitive to uncertainty, suggesting $C_{W}$ will tend to have the largest uncertainty in WEC experiments. Key uncertainty sources in $C_{W}$ will likely be those related to incident wave height and power absorbed by the WEC. WEC power uncertainty arises due to (1) PTO modelling challenges and simplifications (i.e. simple damping mechanisms that simulate prototype PTO systems), (2) power not being measured directly but derived from measured kinematic and dynamic quantities, and (3) the model-prototype power disparity, where power is scaled by the length scale raised to 3.5 (watts at model-scale equate to hundreds or thousands of kilowatts at full-scale). Additionally, non-similitude of Froude and Reynolds Re scaling can introduce uncertainty due to scale effects. To avoid significant viscous effects, Re number should be above critical, which for WEC model tests has been estimated to be $\sim 1 \mathrm{e} 5$ [34. The Re number for this experiment was estimated to be 1.4e5-4.5e5 (see [53]), suggesting for this model Re was a negligible parameter causing scale effects. It is currently not clear how to include uncertainty due to scale effects in, for example, $C_{W}$ uncertainty, which requires a broader experimental uncertainty analysis methodology (elaborated on below).

We found that $u_{B}$ tended to be higher than $u_{A}$. While this may be because $u_{B}$ evaluation is less well defined, $u_{B}$ can in principle be as quantitatively accurate as $u_{A}$. Additionally, given the uncertainty of power-related and loads quantities in irregular waves was similar or slightly smaller than that in regular waves, predictions of prototype power performance and loads might be better based on irregular wave results. Finally, we anticipate that experiments with WECs that have nonlinear motions, power, and loads will show similar uncertainty levels as those reported here.

More generally, this work argues for the value of UA, because when applied in WEC model tests the execution and outcomes can improve considerably. For instance, GUA generates virtual experience of the experiment ahead of time - an integrated grasp of key parameters and uncertainties. These insights can then be used to design test goals, the test matrix, and other technical components [30. These efforts would also identify critical measurements that demand special attention (which the example in subsection 4.6 demonstrated). Special attention might entail choosing higher-accuracy instruments, calibrating instruments with more care more often, redundant instruments, ensuring instrument's physical geometry and fixture negligibly influence the quantity being measured, and prioritising uncertainty evaluation of the measurements [28. Thus GUA confers many benefits by permitting access to otherwise inaccessible information about an experiment before it begins. Once 
the experiment does begin, applying DUA provides the means to monitor the 'health' of incoming data and then ascribes uncertainty to the results when they are reported so they can be assessed.

Furthermore, UA is especially important for WEC model tests because WECs uniquely maximise motions and power but must also survive storms. These characteristics often lead to complex experiments characterised by strong nonlinearities [54, PTO modelling difficulties [28, 9], and scale effects [39, 51 among other experimental challenges [12. Besides this complexity, developers often have tight budgets, resulting in a limited test matrix. And if troubles delay the experiment (to be expected), the test matrix could be further reduced, or facility costs increased. It therefore seems prudent to understand, deeply, the experiment before and as it unfolds. UA is a most effective means to gain such understanding. It also increases the chances of an experiment completed on time, to budget, with high-quality and relevant results that stakeholders can be confident in.

More broadly, while model tests assess a WEC's hydrodynamic energy conversion and loads, a WEC technology consists of many subsystems: hydrodynamic conversion, a PTO/generator, means of survival, energy transformation, offshore operations, environmental aspects, and grid integration 7]. Until a full-scale WEC operates for months or years, these subsystems will contain considerable uncertainty, and the uncertainty will be higher the more inchoate a technology is [26]. To prioritise UA in model tests, then, is to erect strong foundations on which a WEC technology is built. The perceived extra investment may yield a strong return on a technology's commercial viability.

Limitations of the work are the following. The investigation focused on a bottom-fixed OWC WEC with a unidirectional flow PTO, and an experiment typical of TRL 1-4. The examples herein are therefore rather specific to OWC WECs and similar experiments. UA principles, however, are independent of application. So although the work focuses on this unconventional technology, it may nevertheless be informative for future experiments with different WECs. Notwithstanding ITTC's Uncertainty Analysis for a Wave Energy Converter publication, which is good foundation, it is clear that further research is needed to investigate the unique challenges of WEC-specific UA in a range of experiments and WECs, including arrays. For example, point-absorbers [19, 20], terminators, attenuators, and designs with flexible materials.

While we included but a small sample of many UA uses, other references provide other instructive applications [30, 29. With uncertainty propagation we focused on the MCM because it is the preferred method according to [30, and 29] recommends it for applications relevant to WEC experiments characterised by multiple nonlinear, time-dependent quantities. The MCM offers advantages due to its relative ease of implementation especially for phase-averaged quantities (e.g. OWC power) and its superior accuracy compared TSM which linearises the measurement model and thus may provide an inadequate representation. However, this work does not substantiate the claimed advantages of the MCM over the TSM, which would require a comparison of uncertainty results when propagated 
by the MCM and by the TSM. This is reserved for future work. The irregular wave UA may also be considered basic, not comprehensive, so dedicated investigations are needed to address identified irregular wave challenges [55, 56. To a large degree evaluating uncertainty in the power matrix and load matrix, based on a reduced set of wave parameters, is not well understood [57, 58, 44, 26]. Further, extrapolating model scale data and uncertainty to predict prototype performance and risk is a subject in need of further investigation [12].

Finally, this work focused more on measurement uncertainty, rather than experimental uncertainty. Experimental uncertainty includes broader uncertainties such as scale effects and laboratory effects. Future directions may look at developing a fully experimental UA methodology, including the effects of scale and the laboratory.

\section{Conclusion}

General uncertainty analysis facilitates effective experiment planning and design by assuring the right test goals are set, the general uncertainties identified and understood, the right instruments, apparatus, and procedures used, and the right test matrix designed. Detailed uncertainty analysis quantifies the quality of results both during the experiment, by providing the means to monitor apparatus and incoming data and determine whether further measures are needed to reduce the uncertainty, and after the experiment, to ascribe uncertainty to reported results so that those who use the results can assess their reliability, compare them objectively, and use them confidently. It also identifies uncertainty components at a more detailed level. The Monte Carlo method for propagating uncertainty, which can be applied in both general and detailed uncertainty analysis, is a practical alternative to the 'law of propagation of uncertainty' as it provides better accuracy and reduces analysis effort, so it will likely become the preferred method. Overall, uncertainty analysis is an indispensable tool for experimentation.

Uncertainty analysis has already been put to good use in model test experiments of offshore, ship, and coastal structures. As our methodology and results demonstrate, it can also be put to good use in model test experiments of WECs, especially because WECs are more complex systems. Despite its clear benefits, however, uncertainty analysis has largely been overlooked in WEC model tests. We anticipate that if uncertainty analysis is embraced and applied in WEC model tests with our methodology as a template, the execution and outcomes will improve considerably; improvements can also be expected in the commercial viability of a WEC technology. Therefore, uncertainty analysis should be carried out in WEC model test experiments; our methodology demonstrates how to carry it out and its usefulness in identifying, quantifying, and mitigating uncertainties.

The following recommendations are offered to refine international guidelines on uncertainty analysis 


\section{References}

[1] IEA, Total primary energy supply (tpes) by source, world 1990-2017 (2017).

URL https://www.iea.org/data-and-statistics

[2] G. Payne, Guidance for the experimental tank testing of wave energy converters, Tech. rep., The

[3] B. Holmes, Tank Testing of Wave Energy Conversion Systems, European Marine Energy Centre (EMEC) (2009).

[4] B. Holmes, K. Nielsen, Annex II Task 2.1: Guidelines for the development \& testing of wave energy systems, International Energy Agency Ocean Energy Systems (2010).

680

[5] EquiMar, Equimar deliverables (2013).

URL https://www.equimar.org/equimar-project-deliverables.html

[6] MaRINET, Marinet research reports (2016).

URL http://www.marinet2.eu/archive-reports-2/research-reports/ 
[7] A. Pecher, J. Peter Kofoed, Handbook of ocean wave energy, Springer Nature, 2017.

[15] W. Qiu, J. S. Junior, D. Lee, H. Lie, V. Magarovskii, T. Mikami, J.-M. Rousset, S. Sphaier, L. Tao, X. Wang, Uncertainties related to predictions of loads and responses for ocean and offshore structures, Ocean Engineering 86 (2014) 58-67.

[16] ITTC, Ittc recommended guidelines: General guideline for uncertainty analysis in resistance tests (7.5-02-02-02), Proceedings of the 28th International Towing Tank Conference (Recommended Procedures and Guidelines register).

[17] B. Wu, M. Woodward, S. Nishio, A. Olivieri, L. Perez Rojas, M. van Rijsbergen, A. DerradjiAouat, The specialist committee on uncertainty analysis - final report and recommendations to the 26th ittc, Tech. rep., International Towing Tank Conference (2011). 
[29] BIPM, IEC, IFCC, ILAC, ISO, IUPAC, IUPAP, OIML, Jcgm 101:2008: Evaluation of mea-

[19] L. O'Boyle, Assessment of wave basin homogeneity for wave energy converter array studies, Proceedings of the European Wave and Tidal Energy Conference (EWTEC).

[20] P. Lamont-Kane, M. Folley, T. Whittaker, Investigating uncertainties in physical testing of wave energy converter arrays, Proceedings of the European Wave and Tidal Energy Conference (EWTEC).

[21] J. Orphin, J.-R. Nader, I. Penesis, D. Howe, Experimental uncertainty analysis of an owc wave energy converter, Proceedings of the European Wave and Tidal Energy Conference (EWTEC).

[22] J. Orphin, I. Penesis, J.-R. Nader, Uncertainty analysis for a wave energy converter: the monte carlo method, Proceedings of the Asian Wave and Tidal Energy Conference (AWTEC).

[23] E. B. Mackay, A. S. Bahaj, P. G. Challenor, Uncertainty in wave energy resource assessment. part 1: historic data, Renewable Energy 35 (8) (2010) 1792-1808.

[24] E. B. Mackay, A. S. Bahaj, P. G. Challenor, Uncertainty in wave energy resource assessment. part 2: Variability and predictability, Renewable energy 35 (8) (2010) 1809-1819.

[25] O. R. E. Catapult, Wave and tidal energy yield uncertainty reference document, Tech. rep., Catapult, Offshore Renewable Energy (2015).

[26] C. E. Hiles, S. J. Beatty, A. de Andres, Wave energy converter annual energy production uncertainty using simulations, Journal of Marine Science and Engineering 4 (3) (2016) 53.

[27] J. P. Kofoed, A. Pecher, L. Margheritini, M. Antonishen, C. Bittencourt, B. Holmes, C. Retzler, K. Berthelsen, I. Le Crom, F. Neumann, C. Johnstone, T. McCombes, L. E. Myers, A methodology for equitable performance assessment and presentation of wave energy converters based on sea trials, Renewable energy 52 (2013) 99-110.

[28] ITTC, Ittc recommended guidelines: Uncertainty analysis for a wave energy converter (7.5-02-0703.12), Proceedings of the 28th International Towing Tank Conference (Recommended Procedures and Guidelines register).

\footnotetext{
surement data-supplement 1 to the "guide to the expression of uncertainty in measurement"propagation of distributions using a monte carlo method, Joint Committee for Guides in Metrology, JCGM 101.
} 
[30] H. W. Coleman, W. G. Steele, Experimentation, validation, and uncertainty analysis for engineers, 4th Edition, John Wiley \& Sons, 2018. doi:10.1002/9781119417989.

[31] BIPM, IEC, IFCC, ILAC, ISO, IUPAC, IUPAP, OIML, Jcgm 100:2008: Evaluation of measurement data-guide to the expression of uncertainty in measurement, Joint Committee for Guides in Metrology, JCGM 50 (2008) 134.

[32] A. F. O. Falcão, J. C. C. Henriques, Oscillating-water-column wave energy converters and air turbines: A review, Renewable Energy 85 (2016) 1391-1424. doi:10.1016/j.renene.2015.07. 086 .

[33] A. Fleming, G. MacFarlane, S. Hunter, T. Denniss, Power performance prediction for a vented oscillating water column wave energy converter with a unidirectional air turbine power take-off, The 12th European Wave and Tidal Energy Conference (EWTEC) (2017) 1-7.

[34] W. Sheng, R. Alcorn, T. Lewis, Physical modelling of wave energy converters, Ocean Engineering 84 (2014) 29-36. doi:10.1016/j.oceaneng.2014.03.019.

[35] Wave swell energy, Online (2020).

URL https://www. waveswell.com/

[40] D. R. Noble, S. Draycott, T. A. D. Davey, T. Bruce, Design diagrams for wavelength discrepancy in tank testing with inconsistently scaled intermediate water depth, International Journal of Marine Energy 18 (2017) 109-113. doi:10.1016/j.ijome.2017.04.001.

URL https://dx.doi.org/10.1016/j.ijome.2017.04.001 
[51] S. Dai, S. Day, Z. Yuan, H. Wang, Investigation on the hydrodynamic scaling effect of an owc type wave energy device using experiment and cfd simulation, Renewable Energy.

[52] M. R. Eldrup, T. L. Andersen, Applicability of nonlinear wavemaker theory, Journal of Marine Science and Engineering 7 (1) (2019) 14. 
[54] M. Penalba, G. Giorgi, J. V. Ringwood, Mathematical modelling of wave energy converters: A

[53] J. Orphin, I. Penesis, J.-R. Nader, Uncertainty in hydrodynamic model test experiments of wave energy converters, Ph.D. thesis, National Centre for Maritime Engineering and Hydrodynamics, Austalian Maritime College, University of Tasmania (2020). doi:10.13140/RG.2.2.12979. 66088. review of nonlinear approaches, Renewable and Sustainable Energy Reviews 78 (2017) 1188-1207. doi:http://dx.doi.org/10.1016/j.rser.2016.11.137. URL http://www.sciencedirect.com/science/article/pii/S1364032116308784

[55] S. Draycott, B. Sellar, T. Davey, D. R. Noble, V. Venugopal, D. M. Ingram, Capture and simulation of the ocean environment for offshore renewable energy, Renewable and Sustainable Energy Reviews 104 (2019) 15-29. doi:10.1016/j.rser.2019.01.011. URL https://dx.doi.org/10.1016/j.rser.2019.01.011

[56] I. Penesis, W. Batten, A. Fontaine, H. Kyoung Shin, Y. L. Shanghai Jiaotong, M. Kraskowski, P. Andreas Berthelsen, M. Murai, A. Babarit, Specialist committee on hydrodynamics modelling of marine renewable energy devices - final report and recommendations to the 28 th ittc, Tech. rep., International towing Tank Conference (2017).

[57] J.-B. Saulnier, M. Prevosto, C. Maisondieu, Refinements of sea state statistics for marine renewables: A case study from simultaneous buoy measurements in portugal, Renewable Energy 36 (11) (2011) 2853-2865.

[58] R. Pascal, G. Payne, C. Theobald, I. Bryden, Parametric models for the performance of wave energy converters, Applied Ocean Research 38 (2012) 112-124. 